\title{
Tentativo di controllo del periodo sismico siciliano iniziato il 14 Gennaio 1968
}

\author{
(About an attempt to control the sicilian seismir phenomena started \\ on January 14h, 1968)
}

I'. E. VALER

Ricevuto 20 Febluraio 1969

Riassunto. -- Vengono discussi i risultati di un tentativo di controllo del fenomeno sismico siciliano, effettuato sulla base del metodo intrinseco e della teoria dell' "elastic rebound".

SUMMARY. - The results of an attempt to control the sieilian seismice phenomena are discussed. The attempt has been made on the basis of the intrinsic method and of the elastic rehomd theory.

1. - IA TEORIA DELL' GLASTIC REBOUND" SECONDO H. BEXIOFF (*).

In un fenomeno sismico di natura tettonica si possono distinguere generalmente tre fasi e precisamente una serie di scosse premonitorie, la scossa principale e ma serie di repliche.

La spiegazione di questo fatto costituisce un problema difficile e la teoria formulata da $\mathrm{H}$. Beniofr nel 1951 ( $\left.{ }^{1}\right)$ è forse quanto di meglio possa oggi disporre la sismologia, anche perchè può essere utilizzata per un controllo del decorso del fenomeno.

(*) Durante il Convegno Annuale dell Associazione Geofisica Italiana, tenuto a Roma dal 7 al 9 Harzo 1968, l'Autore fece una commicazione sull'andamento del terremoto siciliano (10).

Per cause indipendenti dalla sua volontà non potè tradure, in tempo utile per la pubblinzione sugli Atti del Convegno, la comminicazione in una nota seritta. 
Sostanzialmente la teoria di H. Benioff fonda su due principi, uno di carattere geofisico e l'altro basato sul comportamento reologico delle rocee in laboratorio.

Il principio geofisico, detto dell" elastic rebound ", ronsiste nell'ipotesi che i terremoti tettonici siano dovuti ad una rapida liberazione della deformazione lentamente accumulata nelle masse di roceia, rhe costituiscono ma faglia.

L'altro principio consiste nell'ammettere che il comportamento "in loco n delle rocas possa essere descritto mediante le leggi osservate in laboratorio.

Secondo queste legrgi la deformazione di una roccia in funzione del tempo puo essere espressa da relazioni del tipo

$$
\begin{gathered}
S=A+B \log t \\
S=C+D\left[1-e^{-\alpha\left(t-t_{0}\right)} \beta^{\beta}\right]
\end{gathered}
$$

a seconda che si tratti rispettivamente di deformazioni di pressione (1razione) o di distorsione, e nelle quali $A, B, C, D, t_{0}$, a e $\beta$ sono costanti dipendenti dalla natura del materiale.

Sulla base di questi due principi, H. Benioff formula un modello del mecoanismo, secondo il quale si verifica un fenomeno sismico di ("arattere tettonico.

Il modello è illustrato dalla Fig. 1.

Gli elementi elastici $G_{1}$ e $G_{2}$ e l'elemento resistente $R_{\text {o descrivono }}$ il comportamento delle roce in cui esiste la faglia, mentre $R$, è lelemento secolare resistente del mezzo in cui la tensione greneratrice secolare $F$, supposta costante, si origina e con $F$ determina l'incremento della deformazione degli elementi elastici $G_{1}$ e $G_{2}$.

Si supponga che in seguito ad un fenomeno sismico gli elementi $\left(r_{1}\right.$ e $G_{2}$ siano totalmente rilassati e che la faglia sia saldata.

Il sistema $G_{1}, G_{2}, R_{1}, R_{2}$ viene lentamente deformato e l'incremento della deformazione nel tempo è determinato dalla tensione generatrice secolare $F$ e dal termine secolare resistente $R_{1}$, che si ritiene maggrores di $R_{2}$.

Ia deformazione di $G_{1}$ e $G_{2}$ esercita ma tensione sulla faglia, la quale cresce di un incremento secolare, fino al momento in cui questa tensione supera, in qualche punto, la tensione di coesione esistente nella faglia e inizia in quel punto lo scorrimento della faglia stessa.

Una volta che il movimento sia iniziato, lattrito statico fra le labbra della faglia si trasforma in attrito dinamico e lo scorrimento 


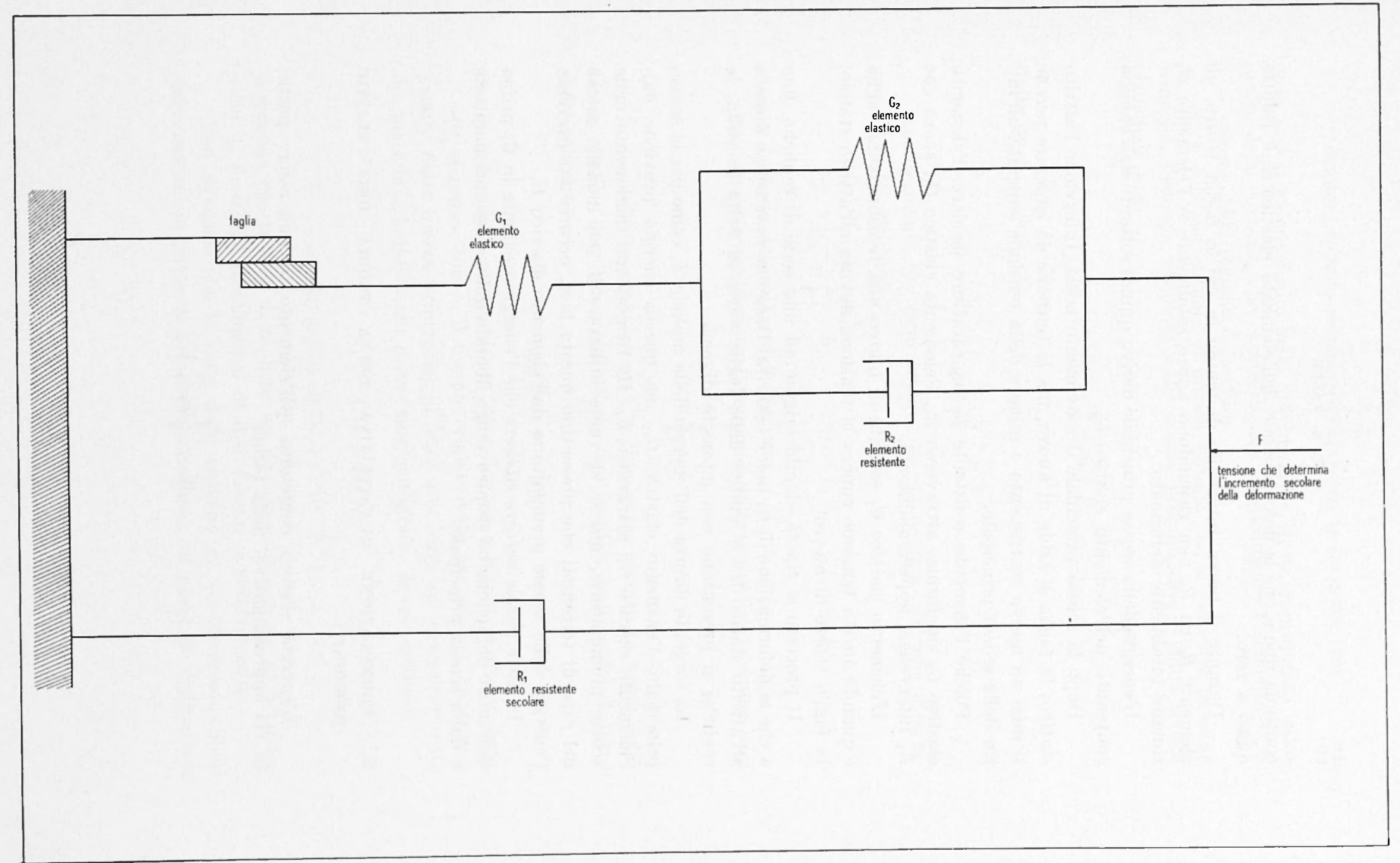

Fig. 1 - Schema del modello di H. Benioff secondo il quale si realizza un fenomeno sismico tettonico. 
continua fino a che la deformazione dellelemento elastico $b_{1}$ ie ridotta quasi a zero.

Dourante il breve intervallo di tempo in cui la fagria scorre, grli elementi $R_{1}$ ed $R_{2}$ non rispondono apprezabilmente e leelemento $r_{2}$ rimane totalmente deformato.

L'energia della scossa principale deriva quindi soltanto dall'energia rontentuta nell'elemento elastico $G_{1}$.

Iopo la scossa principale il movimento cessa. Interviene laatrito statico, la faglia si salda di nuovo, ma la tensione da superare perchè si inizi un nuovo seorrimento è minore della tensione superata all'inizio della scossal principale.

Poichí l'elemento resistente $R_{1}$ ha carattere secolare, l'elemento elastico $t_{2}$ rideforma, attraverso $R_{2}$, l'elemento elastico $G_{1}$, senza che $R_{1}$ intervenga apprezzabilmente.

L'elemento elastico ( $r_{1}$ esereita di nuovo una temsione sulla faglia e quando questa tensione supera la tensione dovuta allattrito statico, la faglia scorre di nuovo.

Il processo si ripete, dando origine ad ma serie di repliche, fino a che la deformazione di $r_{2}$ non è esanuita. Dato che la tensione dovuta allattrito statico fra le labbra della fagria varia di volta in volta, le repliche si presentano con intensità diversal.

Ia sorgente diret ta dell'energia delle repliche è, come per la seossa principale, l'elemento elastico $t_{r_{1}}$, ma questa energia proviene dall'elemento elastieo $r_{2}$, attraverso $R_{2}$. II. Benioff non tiene conto delle scosse premonitorie, ma se si vuole utilizzare il suo modello anche nel caso di teremoti che presentino questa fase, oceorre far derivare l'energia delle scosse premonitorie dall'elemento elastico $G_{1}$.

In altre parole ocoore ritenere che l'energia esistente in $t_{1}$ prima dellinizio del fenomeno sismico venga liberata dalle scosse premonitorie - Clalla scossa principale.

2. - FORMULAZIONE QUANTITATTA DELLA TEORIA DELL'“ FLASTIC BHBO(X)".

L'energia elastion contenuta nell'elemento $G_{1}$ può essere posta, in via approssimativa, nella forma

$$
W_{1}=\frac{1}{2} \mu_{1} \varepsilon_{0} V
$$


dove $\varepsilon_{o} \dot{e}$ il valore medio della deformazione, $\mu_{1}$ un appropriato valor medio delle costanti, che caraterizamo elasticamente l'elemento $a_{1} e$ $V$ il volume della regione deformata.

Se $p$ è la percentuale di questa energia che si converte in onde sismiche, lenergia irmadiata dalle scosse premonitorie e dalla seossa principale sarà

$$
E_{0}=\frac{1}{2} \mu_{1} \varepsilon_{0} \gamma p
$$

dalla quale si trae

$$
\varepsilon_{o}=\left(\frac{2}{\mu_{1} V p}\right)^{1 / 2} E_{0}^{1 / 2}
$$

Analogamente per ogni replica, la cui energia proviene direttamente da $G_{1}$, risulterà

$$
\varepsilon_{j}=\left(\frac{2}{\mu_{1} V p}\right)^{1 / 2} E_{j} \quad\left(j=1,2, \ldots, l_{i}, \ldots, n\right)
$$

dove $"$ i il numero totale delle repliche.

La deformazione liberata dalle repliche alla fine della $k^{m n}$ replica salà

$$
N_{k}=\sum_{1}^{k} \varepsilon_{j}
$$

is possibile determinare questa grandezad, almeno in unitia arbitrarie, percho l'energia irmadiata da ogni replica puo essere dedota dalla relazione

$$
\mathrm{I} \operatorname{sog} E=b+a M
$$

che la lega alla sua magnitudo $M$ e dove $a$ e $b$ somo costanti note.

Se si indica con $t_{k}$ il tempo origine della $k^{m a}$ replica, contato a partice dalla scossa principale, in base alle leggi sul comportamento dei materiali, indicate nel precedente paragrafo, dovlà risultare

$$
\begin{gathered}
S_{k}=A+B \log t_{k} \\
S_{k}=\left(+D+1-e^{-\alpha\left(t_{k}-t_{o}\right) \beta}\right]
\end{gathered}
$$

a seconda che la serie di repliche sia dovuta a liberazione di deformazioni di pressione (trazione) o di distorsione, rispettivamente.

Puo avvenire ('he la serie delle repliche di un terremoto liberi deformazioni in parte di pressione (trazione), in parte di distorsione, 
come mostrano alcuni diagrammi sperimentali costruiti da II. Benioff, che sono risultati in discreto accordo con le precedenti relazioni teoriche.

\section{3. - II, RENDIMENTO ATTUALE DEL PROCFSSO DELLF REPLICHE.}

Si consideri ora l'energia esistente in $r_{r_{2}}$ prima dell'inizio del fenomeno sismico. Indicando con $\mathbb{W}_{2}$ questa energia risulterà

$$
W_{2}=\frac{1}{2} \mu_{2} \varepsilon_{2}^{2} V
$$

dove $\mu_{2}$ e $\varepsilon_{2}$ sono rispettivamente la costante elastica e la deformazione massima dell'elemento $G_{2}$.

Poiché l'energia contenuta in $\left(r_{1}\right.$ vale

$$
W_{1}=\frac{1}{2} \mu_{1} \varepsilon_{0}^{2} V
$$

si ha subito

$$
\frac{W_{2}}{W_{1}}=\frac{\mu_{2} \varepsilon_{2}^{2}}{\mu_{1} \varepsilon_{5}^{2}} .
$$

Dato (he prima dell'inizio del fenomeno sismion le tensioni in $G_{1}$ e (r, sono uguali

$$
\mu_{2} \varepsilon_{2}=\mu_{1} \varepsilon_{0}
$$

consegule

$$
\frac{W_{2}}{W_{1}}=\frac{\varepsilon_{2}}{\varepsilon_{0}}
$$

()ra

$$
\varepsilon_{2}=\left(\frac{2}{\mu_{1} V p}\right)^{1 / 2} \sum_{1}^{n} E_{j}^{1 / 2}
$$

pertanto

$$
\frac{W_{2}}{W_{1}}=\frac{\sum_{j}^{n} E_{j}^{1 / 2}}{E_{0}{ }^{1 / 2}} .
$$

Supposto che il fattore $p$ sia lo stesso per tutte le seosse, che cosi $\mathrm{i}$ tuiscono il fenomeno sismico, indicando con $Q$ l'energia di deformazione trasformata in calore nel processo delle repliche, si ha.

$$
\begin{gathered}
p W_{1}=E_{o} \\
p\left(W_{2}-Q\right)=\sum_{1}^{n} E_{j}
\end{gathered}
$$


dove il termine a secondo membro della seconda delle precedenti indica l'energia totale irradiata in onde sismiche dalle repliche.

Sussiste quindi la relazione

$$
\frac{p\left(++\frac{n}{\Sigma}, E_{j}\right.}{E_{0}}-\frac{\sum_{1}^{n} E_{j}{ }^{1 / 2}}{E_{0}{ }^{1 / 2}}
$$

da rui

$$
\vartheta=\frac{1}{p}\left(E_{0}{ }^{1 / 2} \sum_{1}^{n} E_{j}^{1 / 2} \cdots \frac{n}{1} \cdot E_{1}\right)
$$

Se si pone

$$
Q_{j}=\frac{1}{p}\left(H_{0}^{1}{ }^{1 / 2} E_{j}{ }^{1 / 2}-E_{J}\right)
$$

la quantita d'energia dissipata in calore dopo la $k^{m a}$ replica sarà

$$
()_{k}=\frac{1}{p} \sum_{1}^{k}\left(E_{0}{ }^{1 / 2} E_{j}{ }^{1 / 2}-E_{j}\right)
$$

E possibile quindi definire il rendimento attuale del processo delle repliche, rioè il rendimento relativo al passagrgio dellenergia da $f_{2}$ e $r_{1}$ dopo la $k^{m n}$ replical, mediante la relazione

$$
\eta_{k}=\frac{\frac{1}{p} \sum_{j}^{k} E_{j}}{Q_{k}+\frac{1}{p} \sum_{1}^{k} E_{j}}
$$

ossia

$$
\eta_{k}=\frac{\sum_{1}^{k} E_{j}}{E_{n}^{1 / 2} \sum_{1}^{k} E_{j}{ }^{1 / 2}} .
$$

Evidentemente l'energia perduta dall'elemento $\dot{r}_{2}$ dopo la lima replica è data da

$$
\frac{1}{p} E_{o}{ }^{1 / 2} \sum_{1}^{k} E_{j}^{1 / 2}
$$

eosicche il diagramma di H. Benioft non solo rappresenta in muità arbitrarie l'andamento della deformazione liberata dalle repliche in 
funzione del tempo, ma anche, a meno di un fattore, forse assai prossimo ad uno, l'energia perduta dall'elemento $G_{2}$ dopo la $k^{m a}$ replic‘a.

Le repliche resseranno quando

$$
W_{2}-\frac{1}{p} E_{o}{ }^{1 j_{2}} \sum_{1}^{k} E^{j 1 / 2}=0
$$

e ció si verificherì per $k=n$, dove $n$ ì il numero totale delle repliche.

conviene normalizzare le energie ponendo

$$
x_{j}=\frac{E_{j}}{E_{0}}
$$

dove $E_{n}$ i la somma delle energie irradiate in onde sismiche dalle scosse premonitorie e dalla scossa principale e porre

$$
S_{k}=\stackrel{\Sigma}{L}_{1}^{k} x_{j}^{1 / 2}
$$

Per quanto riguarda la deformazione liberata dalle scosise premonitorie e dalla scossa principale, coerentemente con quanto è stato esposto, essisa dovrà essere calcolata mediante la relazione

$$
S_{k_{0}}=\left(\sum_{1}^{k_{j}} x_{j}\right)^{1 / 2}
$$

e risulterì

$$
s_{k_{0}}=1
$$

per $k_{0}=n_{o}$, dove $n_{n}$ è il numero totale delle scosse premonitorie, principale inclusa.

4. - IA DEFORMAZIONE LIBERATA DAL TERREMO'TO SICILIANO DEL 15 Gennaio 1968 in funzione del tempo.

Uno studio dettagliato degli effetti macrosismici del terremoto siciliano del 15 Gennaio 1968 è stato recentemente pubblicato da M. De Panfilis e L. Marcelli ( $\left.{ }^{2}\right)$.

In questa nota ̀̀ quindi sufficiente ricordare che il fenomeno sismico ha presentato le tre fasi caratteristiche e cioè una serie di scosse premonitorie, la scossa principale e una serie di repliche, ancora oggi non totalmente esaurite. 
Lintervallo di tempo intercorso tra la prima scossa premonitoria e la scossa principale è stato molto breve. Si tratta di circa 13 ore. La regrione interessata dal terremoto è illustrata dalla Figr. 2, nella quale sono indicati gli epicentri, individuati dal B.C.I.S., della più forte scossa premonitoria, della scossa principale e delle tre repliche di maggiore intensità.

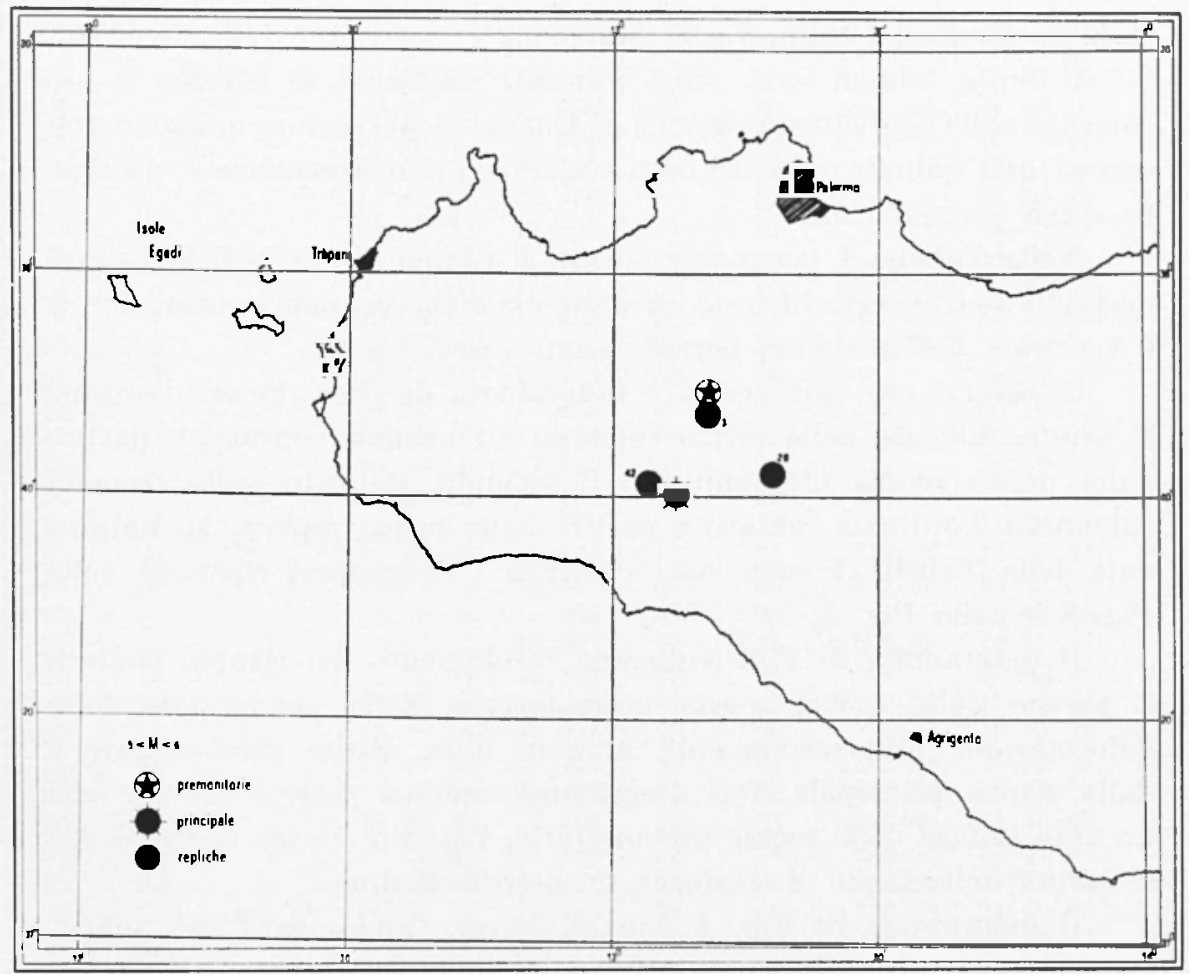

Fig. 2 - Terremoto siciliano del 15-1-1968. - Epicentri della scossa premo. nitoria più intensa, della scossa principale e delle tre repliche più intense.

Vi sono ragioni per ritenere che gri ipocentri delle scosse siano localizzabili nella crosta terrestre e quindi la loro profondità non supera probabilmente una quindicina di $\mathrm{km}$, dato che tale $\dot{\mathrm{e}}$ mediamente l'ordine di grandezza del suo spessore nel Mediterrameo CentroOccidentale $\left({ }^{3}\right)$.

Le sole magnitudo delle scosse di cui si poteva disporre durante il periodo sismico sono state misurate da L. Marcelli nell'Osservatorio sismico di Roma, che dista dalla zoma epicentrale circa $450 \mathrm{~km}\left({ }^{10}\right)$. 
(ili strumenti dellogsservatorio hamono registrato ron sufficiente rhiarezara le scosse di magniturlo nom inferiori a lre, e quindi in questa ricerca non vengono prese in romsiderazione le basse energie.

Isenergia liberata in onde elastiche in ogni scossa is stata callrolata ron la relazione

$$
\log E=9,154+2.145 .11
$$

stabilita da l). I)i Filipluo o. Is. Marrecelli (').

I tempi origine somo slati valutati mediante le tabelle di uso rorrente nellosservatorio Sismico di Roma. Il loro errore massimo nom supera man quindicina di secondi e questa approssimazione i da ritenere piir che sufliciente.

Sella Tabella I vengono riportati il tempo origine e il logarimo decimale dellenergia liberata in onde elastiche da ogni scossa, che si ¿̀ verificata dall’inizio del periodo sismico al 1-IX-68.

Si osservi rhe ogni scossa ó individuata da due numeri doordine. 11 primo, indicato nella prima colomma, i loordinale contato, a partire dalla prima scossa premonitoria, il secomdo, indicato nella secondat colomat o l'ordinale contato a partire dalla prima replica. Mediante i dati della Tabella 1 somo stati rostruiti i diagrammi riportati nella Fig. 3 e nella Fig. 4.

Il diagramma di Fig. 3 mostia l'andamento del tempo, contanto a partire dalla prima scossa premonitoria, della percentuale della deformazione progressivamente liberala dalle scosse premonitorie a dalla scossa principale. Dal rlagramma sembra potersi pilevare che durante la fase delle scosse premonitorie, lattrito statico esistente fra le labbua della faglia si trasforma in attrito dinamico.

Il diagramma di Fig. 4 mostra invece l'andamento nel tempo, contato a partire dalla scossa principale, della pereentuale della deformazione liberata progressivamente dalle repliche, fino al 1 settembre 1968. Sostanzialmente il diagramma di Fig. 4 roincide col diagramma di 11 . Benioff e mostra che la deformazione liberata dalle repliche erat di prosisione (trazione).

Ia Tabella II è riassuntiva. Da questa tabella risulta rhe il maporto fra l'energia contenuta inizialmente in $G_{2}$ o $G_{1}$, alla data 1 Settembre 1968 risultava

$$
\frac{W_{2}}{W_{1}}=1,556
$$

mentre l'energia immliata in onde elastiche dalle repliche alla stessa 
'Tabolla I

\begin{tabular}{|c|c|c|c|c|c|c|c|c|c|}
\hline$k_{0}$ & $k$ & Data & $\begin{array}{c}M \\
\text { (I.M.E.C.) } \\
h \quad m \quad s\end{array}$ & $\begin{array}{c}\operatorname{Jog} E \\
(\mathrm{E} \text { in } \operatorname{cog}(\mathrm{g})\end{array}$ & $k_{0}$ & $k$ & Data & 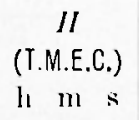 & 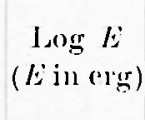 \\
\hline 1 & & 14.1 .188 & 122820 & $19 \cdot 2+5$ & 46 & 38 & .) 1 - (6) & 131830 & $168 \times 3$ \\
\hline 2 & & $"$ & $12 \quad 11 \quad 13$ & 17,098 & 47 & 39 & $2 \cdot-1-(; 8$ & 213810 & 16.883 \\
\hline 3 & & $"$ & $12+350$ & 17,205 & 48 & 40 & $23-1.68$ & 162924 & 16,883 \\
\hline 4 & & $"$ & 131537 & 19,395 & 49 & 41 & " & 165718 & 16,883 \\
\hline 5 & & $"$ & 154820 & 19,760 & 50 & 42 & $25-1-68$ & (0) 5649 & 21,392 \\
\hline 6 & & $"$ & 23 I8 50 & 17,650 & 51 & 43 & $"$ & $1+3532$ & 19,202 \\
\hline 7 & & $15-1-68$ & () & $21,52 \mathrm{I}$ & 52 & 44 & $2(j-1-6 k$ & $0802 \quad 24$ & 18,193 \\
\hline 8 & & " & $(120102$ & 21,959 & 53 & 45 & $27-1-138$ & 174359 & 17,313 \\
\hline 9 & 1 & $"$ & $(0,30.356$ & 17,935 & 54 & 46 & $28-1-68$ & $22 \quad 21 \quad 20$ & $16,8 \times 3$ \\
\hline 10 & 2 & $"$ & 0.31350 & 17,871 & 5i) & 47 & $30-1-68$ & 185048 & 16,239 \\
\hline 11 & 3 & $"$ & $0.3 \quad 18 \quad 30$ & 19,975 & 56 & 48 & , & Is 5I 56 & 16,239 \\
\hline 12 & 4 & " & $\left(\begin{array}{lll}09 & (13 & 55\end{array}\right.$ & 17,849 & 57 & 49 & 1-2-68 & (0) 1657 & 16,239 \\
\hline 13 & 5 & $"$ & $09+020$ & 17,549 & 58 & 50 & " & (1) & 16,239 \\
\hline 14 & 6 & $n$ & 101030 & $17,7+2$ & 59 & 51 & ” & $22 \quad 59 \quad 34$ & 16,024 \\
\hline 15 & 7 & $"$ & $1025 \quad 10$ & 18,193 & 60 & 52 & $2-2-68$ & 142557 & 16.024 \\
\hline 16 & 8 & $"$ & $10+130$ & 16,905 & 19 & 53 & $"$ & 172011 & 16,583 \\
\hline 17 & 9 & $"$ & $1054+4$ & 18,515 & 62 & 54 & $5-2-68$ & $11 \quad 1731$ & 16,883 \\
\hline 18 & 10) & $"$ & $13+130$ & 18,558 & 63 & 55 & $8.2-68$ & $1+4236$ & 17,098 \\
\hline 19 & 11 & $n$ & 13435 & $16,905)$ & 64 & 56 & " & 230240 & 16,132 \\
\hline 20 & 12 & $"$ & 145934 & 18,687 & (j)5 & 57 & $10.2-68$ & 123107 & 16,132 \\
\hline 21 & 13 & $n$ & 151454 & 16,883 & 66 & 58 & " & $20 \quad 02 \quad 17$ & 16,346 \\
\hline 22 & 14 & $"$ & 15374 & 18,429 & 67 & 50 & $12-2-68$ & 112544 & 16,239 \\
\hline 23 & 15 & $"$ & $1539+4$ & 17,914 & (is & 60 & $n$ & 162538 & 19,030 \\
\hline 24 & 16 & $n$ & 163431 & 17,742 & 69 & (i) & $13-2-68$ & $22 \quad 32 \quad 22$ & 17,313 \\
\hline 25 & 17 & $"$ & 164851 & 16,883 & 70 & 62 & $16-2-68$ & $(05) 5201$ & 16,346 \\
\hline 26 & 18 & $"$ & 173131 & 18,408 & 71 & 63 & $2-3-68$ & $21+532$ & 16,454 \\
\hline 27 & 19 & $"$ & $18 \quad 2239$ & 19,159 & 72 & 64 & $4-3-138$ & 233735 & 18,171 \\
\hline 28 & 20 & $"$ & $2219+1$ & 18,472 & 73 & (5.5) & $5.3-68$ & 093437 & 16,024 \\
\hline 29 & 21 & $16.1-68$ & (1) 55351 & 18,193 & 74 & $66 j$ & $15-3-68$ & $21 \quad 4609$ & 16,883 \\
\hline 30 & 22 & " & $(1+0904$ & 17,227 & 75 & 67 & $30-3-68$ & 093537 & 10,883 \\
\hline 31 & 23 & $"$ & 12 । 10. & 16,863 & 76 & 68 & " & 133051 & 16,239 \\
\hline 32 & 24 & $"$ & 131029 & 18,450 & 77 & 69 & $"$ & 133743 & $19,(15) 2$ \\
\hline 33 & 25 & $"$ & $1+2923$ & 17,826 & 78 & 70 & $"$ & $1616+7$ & 16,883 \\
\hline 34 & 26 & $"$ & 164237 & 21,542 & 79 & 71 & $1.4-68$ & $161+01$ & $18.3 \times 6$ \\
\hline 35 & 27 & $"$ & $1902 \quad 17$ & 16,905 & 80 & 72 & (j-4-68 & (12 1937 & 16,239 \\
\hline 36 & 28 & $"$ & $2032(1-4$ & $16,9(1)$ & 81 & 73 & $29-5-(58$ & 003657 & 16,6068 \\
\hline 37 & 29 & $17-1-68$ & (1) 362 & $16,9(15)$ & 82 & 74 & " & 013619 & 17,098 \\
\hline 38 & 30 & n & 034019 & 16,883 & 83 & 75 & $3(1)-5.68$ & 785 & 16,239 \\
\hline 30 & 31 & $n$ & 12063 & 16,883 & 84 & 76 & " & 231947 & 18,171 \\
\hline 40 & 32 & $19-1-68$ & 122024 & 17,313 & 85 & 77 & $1-6-68$ & 152937 & I6, 6068 \\
\hline 41 & 33 & $n$ & 22330 & 17,313 & 86 & 78 & $7-6-638$ & (1) $4+2+7$ & $16,0,24$ \\
\hline 42 & 34 & $"$ & $23+2$ & 16,905 & 87 & 79 & $16-8-68$ & $0327 \quad 17$ & $16,88: 3$ \\
\hline 43 & 35 & $20-1-68$ & $(1328 \quad 25$ & 17,098 & 88 & 80 & $1-9-68$ & (05) 0139 & 17,957 \\
\hline 4 & 36 & " & $1934+4$ & 17,313 & 89 & 81 & " & $125+12$ & Ii, (itis \\
\hline 45 & 37 & $21-1-(38$ & $0238 \quad 36$ & 17,935 & & & & & \\
\hline
\end{tabular}




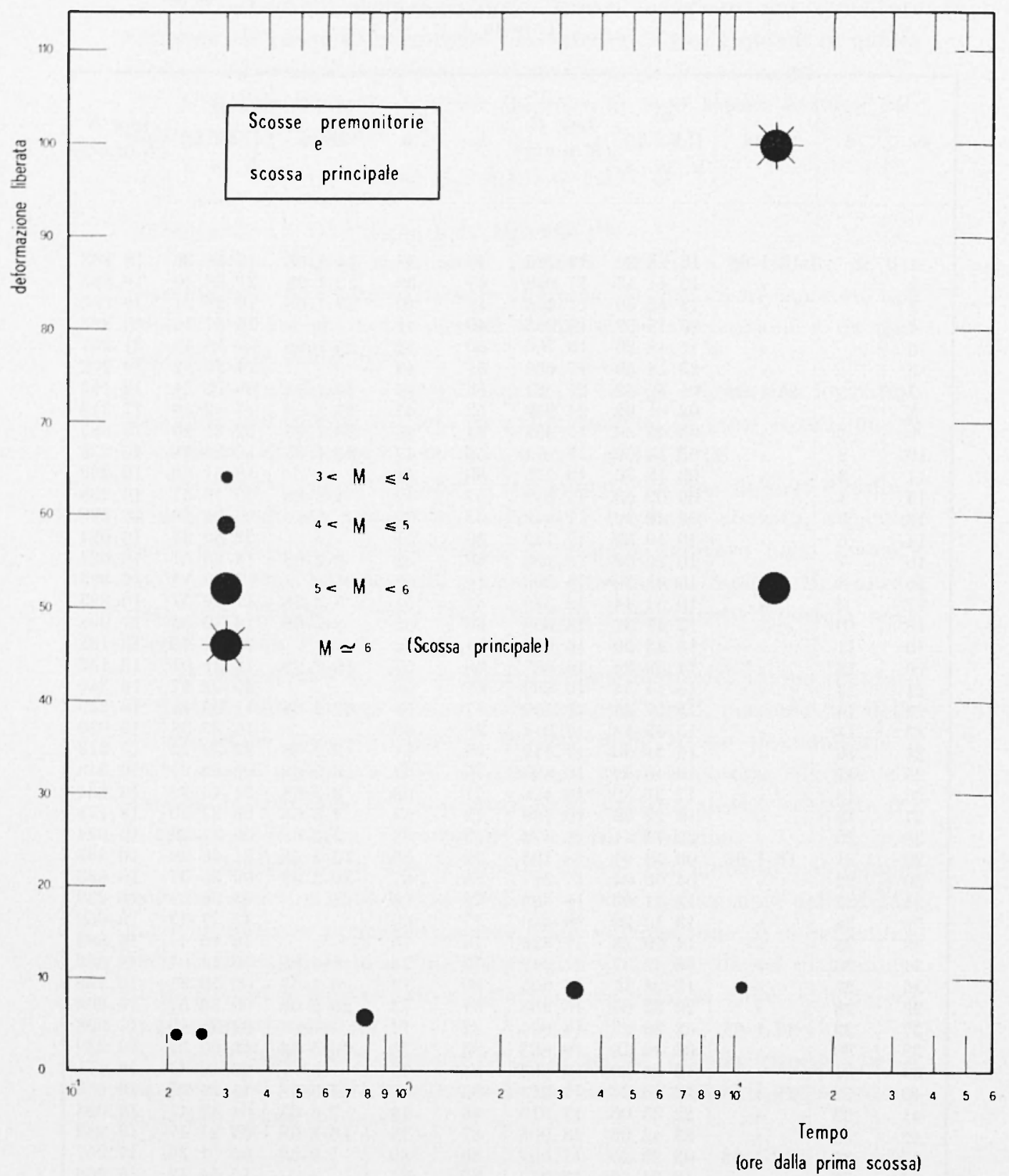

Fig. 3 - Terremolo siciliano del 15-1-1968. -- Percentuale della deformazione liberata dalle scosse premonitorie e dalla scossa principale in funzione del tempo. 
data era solo il $49^{\circ}$, dell'energia irradiata in onde elastiche dalle scosse premonitorie e dalla scossa principale.

Tabella 11

\begin{tabular}{|c|c|c|c|c|}
\hline $\begin{array}{c}\text { Natura } \\
\text { delle scosse }\end{array}$ & Magnitudo & Numero & $\begin{array}{l}\text { Percentuale } \\
\text { dellenergia } \\
\text { liberata } \\
\text { in onde } \\
\text { elastiche }\end{array}$ & $\begin{array}{l}\text { Percentuale } \\
\text { della deforma- } \\
\text { zione liberata } \\
\text { in onde } \\
\text { elastiche }\end{array}$ \\
\hline Premonitorie & $\begin{array}{l}3<. I \leqslant 4 \\
4<. I \leq 5 \\
5<. I \leqslant 6\end{array}$ & $\begin{array}{l}3 \\
3 \\
1\end{array}$ & 27,3 & 52,3 \\
\hline Principale &.$y \cong 6$ & 1 & 72.7 & - \\
\hline Totali & & 8 & 100 & 100 \\
\hline Replicho & $\begin{array}{l}3<y \leqslant 4 \\
4<. y \leq 5 \\
5<. I \leqslant 6\end{array}$ & $\begin{array}{r}54 \\
24 \\
3\end{array}$ & 49,0 & 155,6 \\
\hline Totali & & 81 & $+9,0$ & 155,6 \\
\hline
\end{tabular}

I) rendimento del processo delle repliche risultava pari a

$$
\eta=\frac{49}{15 \tilde{5}, 6}
$$

ossia pari a circa il $31,5 \%$, cosicehé il $68,5 \%$ dell'energia erogata da $G_{2}$ al I Settembre 1968 si era dissipata in calore.

\section{5. - LA DISTRIBLZIONE DEILE SCOSSE NEI, TEMPO.}

La distribuzione delle scosse di un fenomeno sismico nel tempo è una questione che si trascina da più di mezzo secolo e ancora oggi risente dell'errata impostazione iniziale.

Questa impostazione consiste nella ricerca dell'andamento nel tempo della frequenza delle scosse, intendendo per frequenza il 
numero delle scosse che si verificano in un prefissato intervallo di tempos.

Ora non esiste alcun oriterio per stabilire l'ampiezza di questo intervallo e quindi la frequenza delle scosse non è ma grandeyza fisica, perche $n o n$ ammetie ma definizione operativa.

Non i questa la sede per discutere se la nozione di tempo sia stata inventata perehe in natura esistono gli orologi o viceversa, ma è forse conveniente richiamare lattenzione sul fatto che ogni fenomeno naturale ha un tempo proprio, il quale is operativamente definito dal mumero doordine degli eventi in eni il fenomeno si realiz\%a.

Se fra questo numero e il corrispondente numero d'ordine degrli eventi indicati da un'orologio convenzionale esiste ma qualche relazione, essa puo essere messa in evidenza mediante il confronto delle

- due serie di numbri, avendo cura che le due serie abbiano la stessat origine.

In pratica si tratta semplicemente di costruire, con scale opportune, un grafico riportando in ascisse ma delle due serie e l'altra in ordinate.

Per il fenomeno sismico siciliano is stato costruito il diagramma li Fig. 5 .

In ascisse i riportato, in scala logaritmica e misturato in ore, il tempo di ciascuna scossa, contato a partire dalla prima, e in ordinate il relativo numero d'ordine, rontato anch'esso a partire dalla stessa origine.

Il diagramma mostra che il numero d'ordine delle scosse premonitorie d praticamente proporzionale al logaritmo del tempo e altrettanto avviene per le repliche, ma ron andamento differente.

Si rileva facilmente dal diagramma che la scossa principale divide eflettivamente due fasi assai diverse dello stesso fenomeno.

(io) non sembra essere casuale. Infatti gli analoghi diagrammi di Fig. 6 costruiti per il terremoto di Palombara Sabina del 24 Aprile $1901\left(^{5}\right)$ a per $^{2}$ il terremoto di Termini Imerese dell'11 Settembre $1906\left({ }^{8}\right)$, mostrano caratteristiche assai simili.

Oecorre pero avvertire che per il terremoto Marsicano del 13 Gennaio 1915 la dipendenta del numero d'ordine delle repliche dal tempo appare alquanto piò complicata (7-8).

Questo terremoto, a quanto è dato di conoscere, iniziò direttamente con la scossa principale ed ebbe più di milleduecento repliche.

Non sembra peraltro rhe sia stato cansato da scorrimento di faglie. 


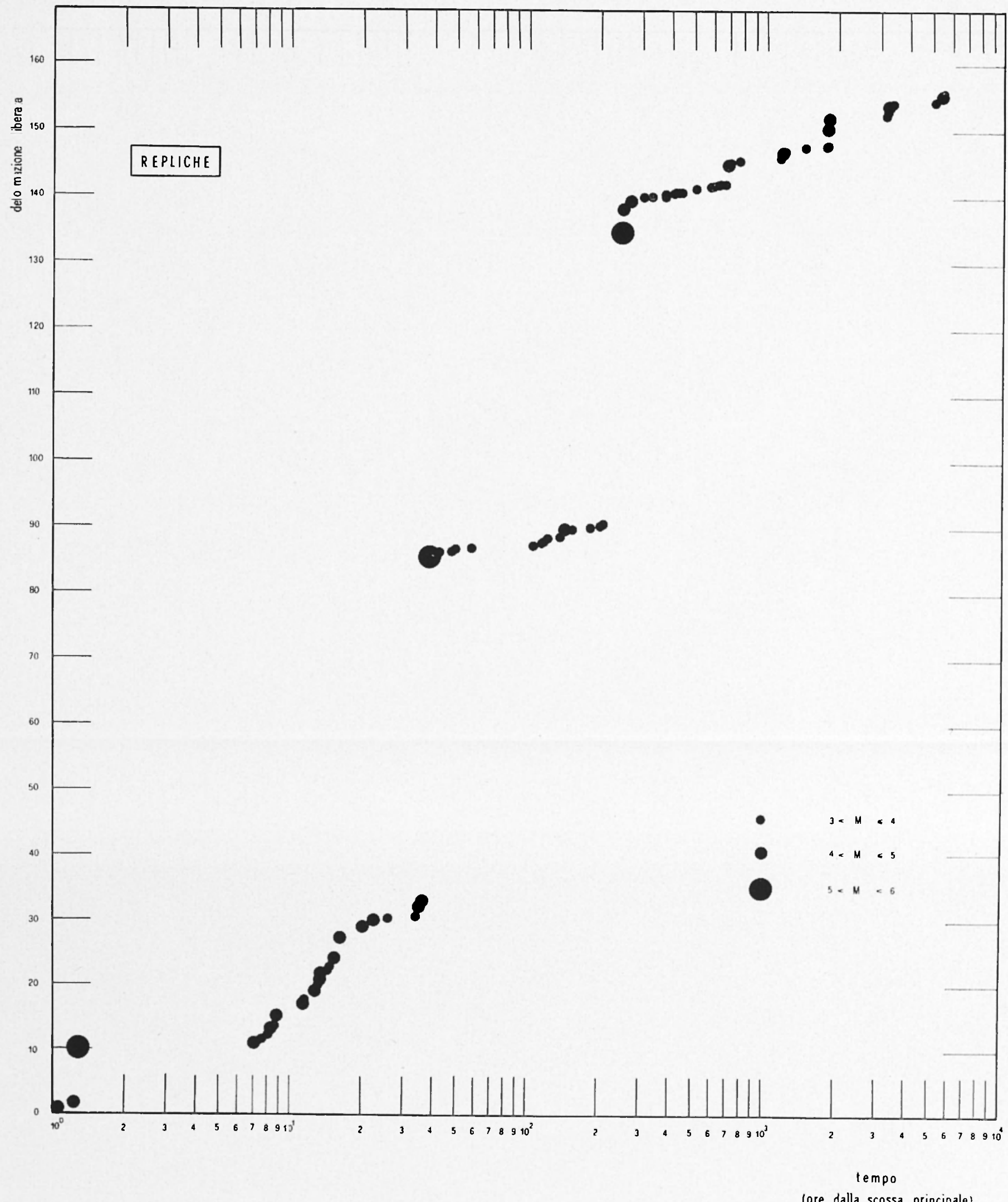

Fig. 4 - Terremoto siciliano del 15-1-1968. - Percentuale della deformazione liberata dalle repliche in funzione del tempo. 
P. E. VALLE

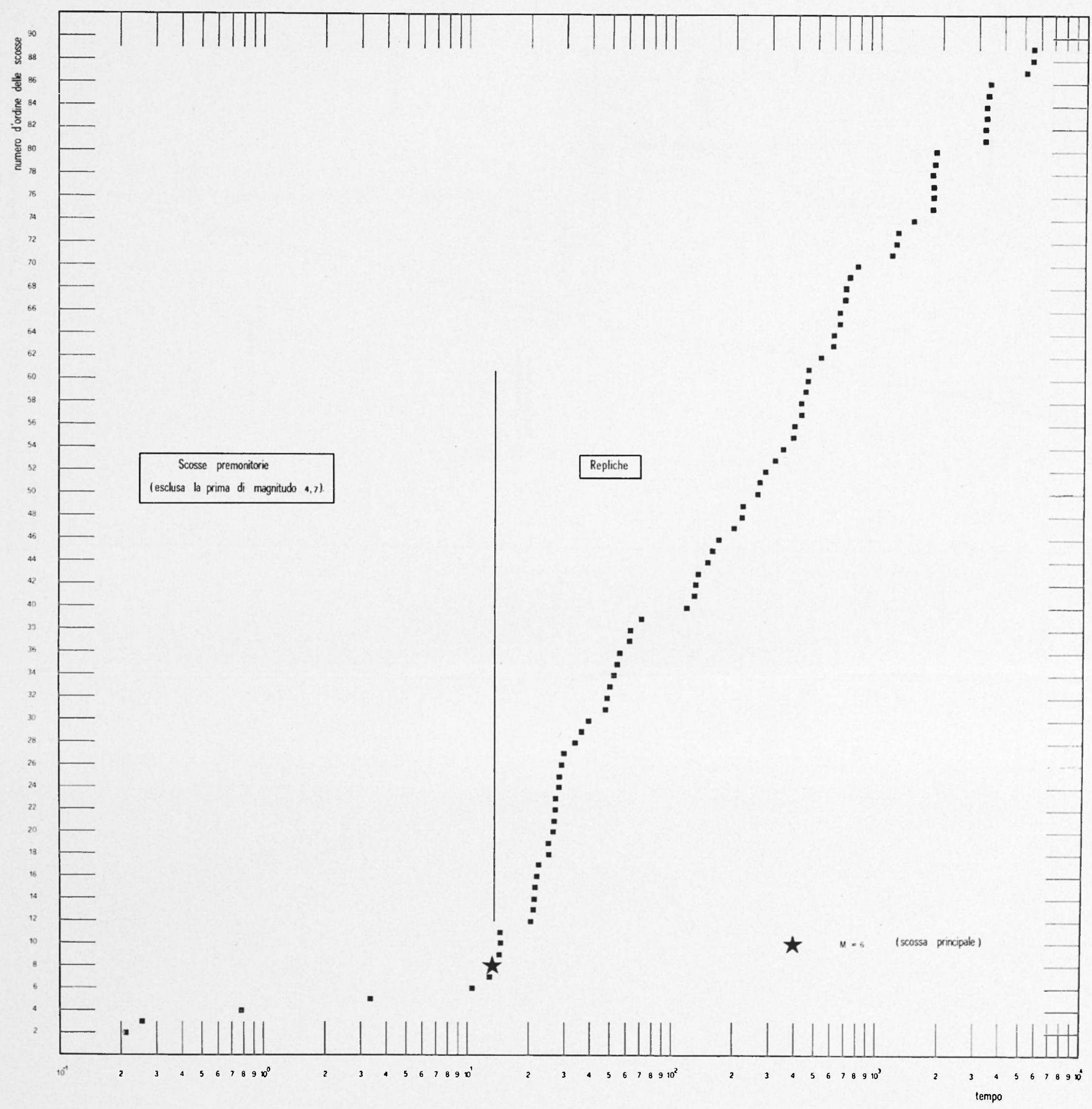

(ore dalla prima scossa)

Fig. 5 - Terremoto siciliano del 1:j-1-1968. - Numero d'ordine delle scosse in funzione del tempo. 


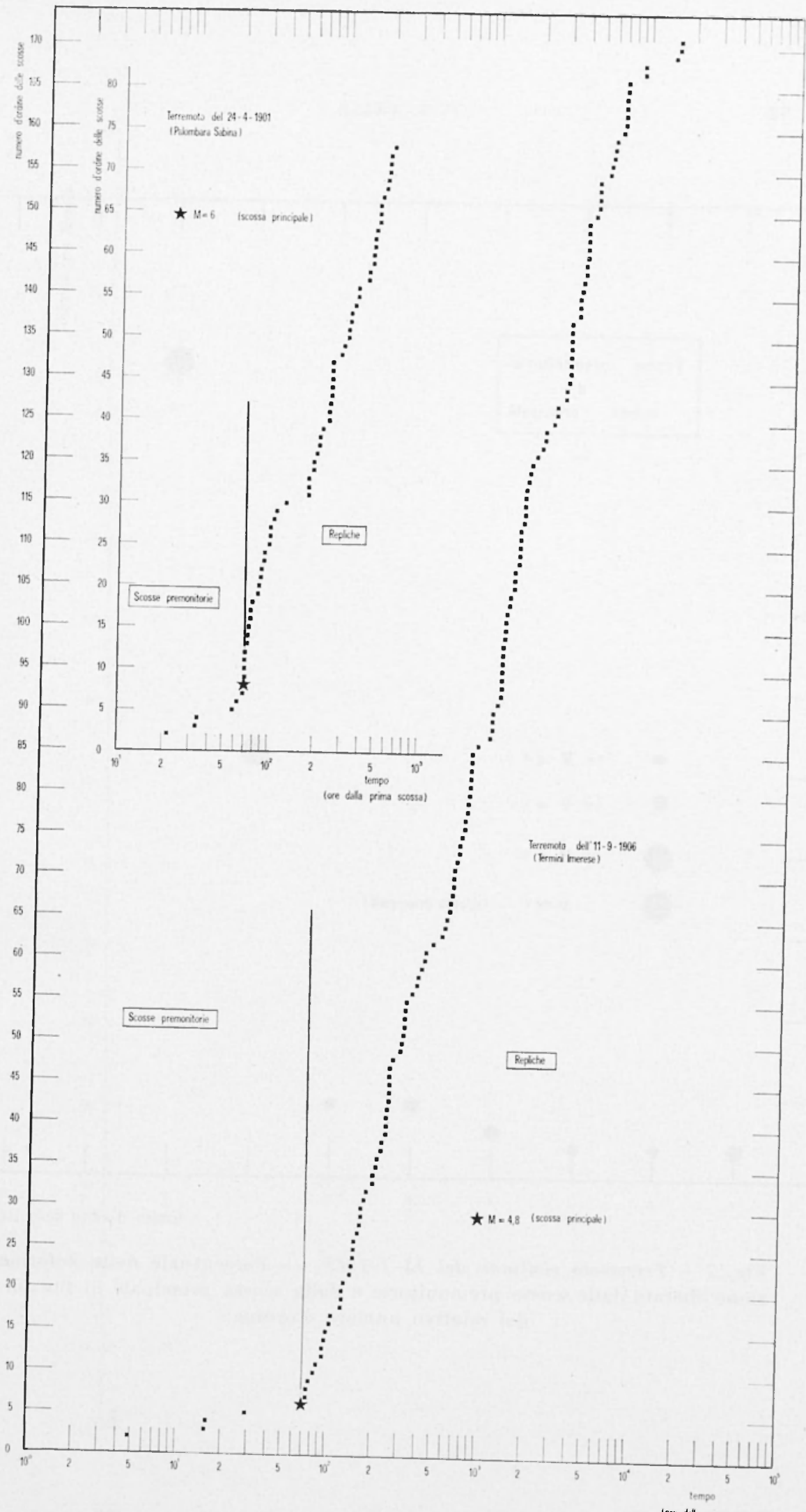

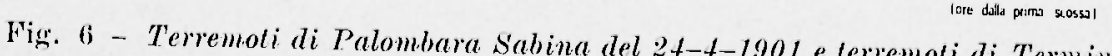
Imerese dell'11-9-1906. - Numero d'ordine delle seosse in funzione del tempo. 


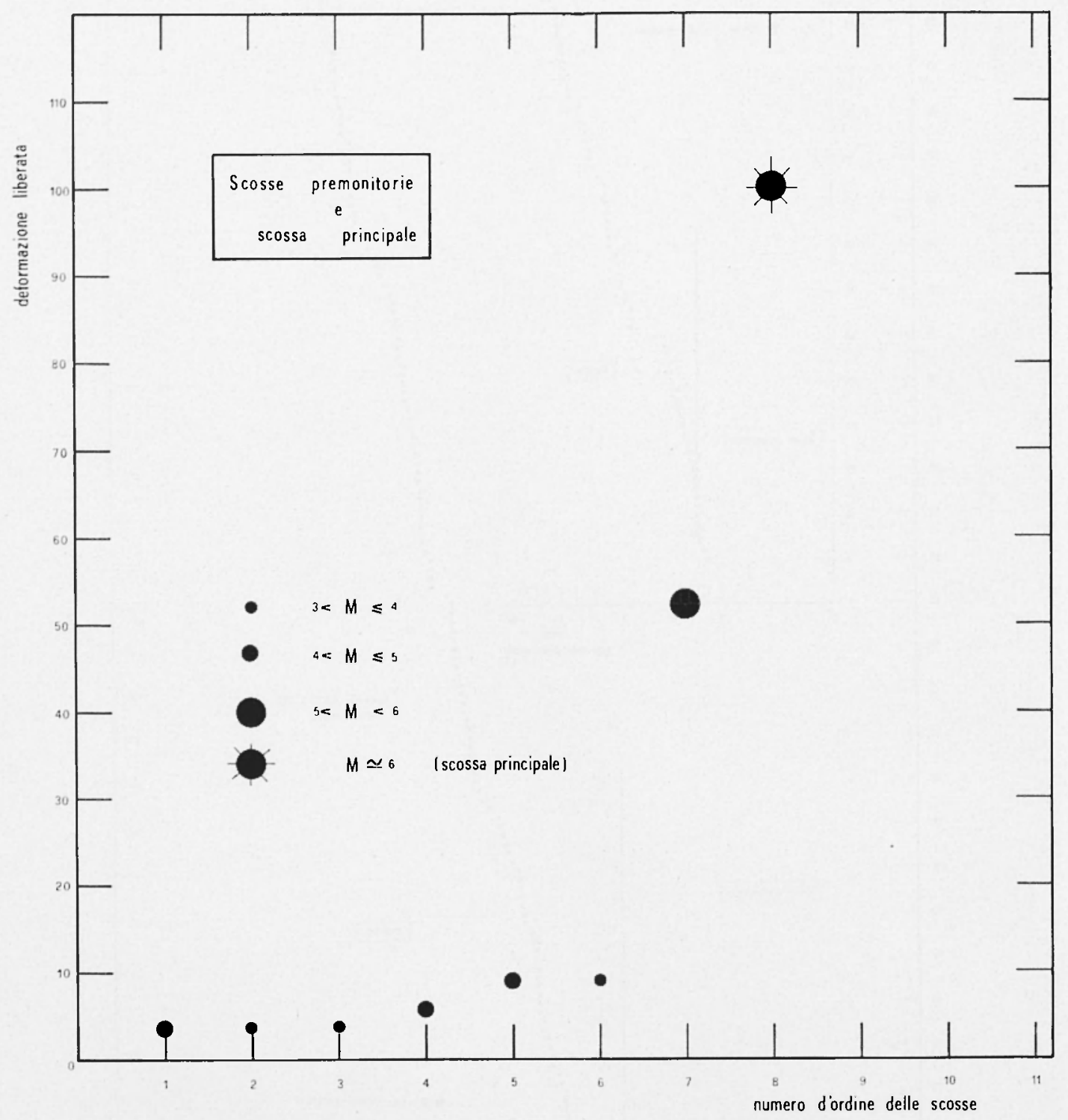

Fig. 7 - Terremoto siciliano del 15-1-1968. - Percentuale della deformazione liberata dalle scosse premonitorie e dalla scossa principale in funzione del relativo numero d'ordine. 


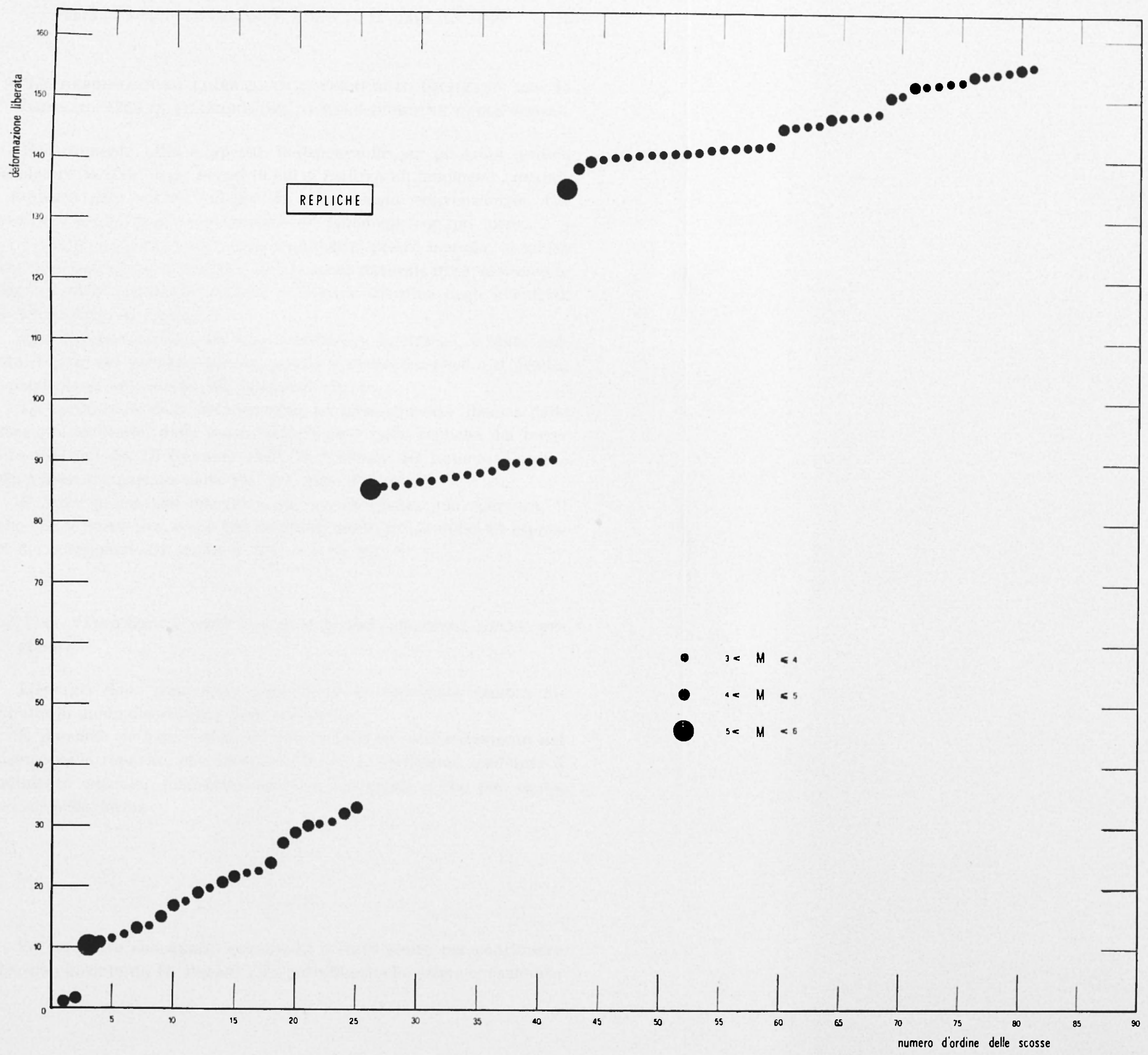

Fig. 8 - Terremoto siciliano del 15-1-196s. - Percentuale della deformazione liberata dalle repliche in funzione del relativo numero d'ordine. 
6. - IA DEFORMAZIONE IIBERATA DAL TERRFAOTO SICILIANO DEI, 15

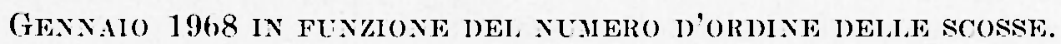

ì rertamente utile e, spesso, indispensabile per gli scopi pratici, ronsiderare la serie degli eventi in cui si realizza un fenomeno naturale in rapporto agli eventi indicati da un orologio convenzionale. Cio significa sostanzialmente confrontare un fenomeno con un altro.

Talvolta peraltro è più utile tralasciare questo metodo, in veriti piuttosto compliato, e studiare un fenomeno naturale in sè, assumendo come variabile temporale propria il numero d'ordine degli eventi in cui il fenomeno si realizza.

Con questo metodo, che si può chiamare intrinseco, ¿̀ stato esegruito il resto del presente lavoro, perchè è molto semplice e si presta. in particolare, allo studio dei fenomeni sismici.

La percentuale della deformazione progressivamente liberata dalle scosse premonitorie, dalla scossa principale e dalle repliche del terremoto siciliano del 15 Gennaio 1968, in funzione del numero dordine delle scosse, è riportata nella Fig. 7 e nella Fig. 8.

E forse prematuro discutere qui questi grafici, ma, per ora, il meno che si possa dire, è che essi risultano molto più semplici ed espressivi dei corrispondenti grafici di Figr. 5 e di Figr. 6.

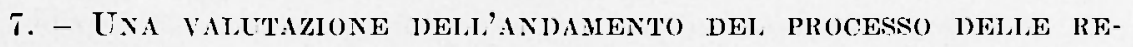
PI.ICHE.

L'energia della serie delle repliche di un terremoto sembra distribuita in modo disordinato nella serie stessa.

È possibile però pervenire ad una definizione dell'andamento del processo delle repliche, che successivamente si verificano, mediante il rendimento attuale, introdotto nel terzo paragrafo e che può essere espresso nella forma

$$
\eta_{K}=\frac{\sum_{j}^{k} x_{j}}{\sum_{1}^{k}-\cdots} x_{j}^{1 / 2}
$$

Va osservato che questo parametro è stato scelto per continuare il discorso iniziato da H. Benioft. Ma potrebbe anche essere considerato 
al di fuogi di tale discosso, rimanendo tuttavia una grandeza valida perche definita operativamente, senza avere peraltro il significato fisico che la teoria di II. Benioff grli conferisce.

Per semplicità conviene porre

$$
\sum_{1}^{k} x_{j}=a_{k}, \quad \sum_{1}^{k} x_{j}^{1 / 2}=b_{k}
$$

e scrivere

$$
\eta_{k}=\frac{a_{k}}{b_{k}} .
$$

Si supponga verificata la $k^{m n}$ replica e di aver calcolato il rapporto $\eta_{k}$.

Dopo l'eventuale successiva $(k+1)^{\text {ma }}$ replica tale rapporto assumera il valore

$$
\eta_{k+1}=\frac{a_{k}+x_{k+1}}{b_{k}+x_{k+1}^{1 / 2}}
$$

dove $x_{k+1}$ è l'energia (normalizzata) liberata in onde elastiche dalla eventuale $(k+1)^{m a}$ replical.

Il rapporto in questione varierà pertanto di

$$
\Delta \eta_{k+1, k}=\frac{x_{k+1}^{1 / 2}\left(x_{k+1}^{1 / 2}-\eta_{k}\right)}{b_{k}+x_{k+1}^{1 / 2}} .
$$

Si vede subito ohe l'andamento del gendimento attuale in funzione del numero dordine delle repliche, contato a partire dalla prima, consente di definire il processo delle repliche stesse come decrescente, stazionario o rrescente a seconda che risulti

$$
1 \eta_{k+1}<0, \quad \Delta \eta_{k+1}=0, \quad \quad 1 \eta_{k+1}>0
$$

perchò queste tre eventualità si verificano se sono rispettivamente soddisfatte le condizioni

$$
x_{k+1}^{1 / 2}<\eta_{k}, \quad x_{k+1}^{1 / 2}=\eta_{k}, \quad x_{k+1}^{1 / 2}>\eta_{k}
$$

nelle quali con $x_{k+1}$ si è indicata la deformazione liberata nella $(k+1)^{m a}$ eventuale replica.

Se quindi nel corso del verificarsi delle repliche, si costruisce un grafico riportando in ascisse il loro numero d'ordine e in ordinate il corrispondente valore del rendimento attuale, si può seguire il fenomeno nelle sue fusi decrescenti, stazionarie e crescenti. 
Si osservi che qualunque sia stato l'andamento del processo alla $k^{m a}$ replica, indicando ron

$$
x_{u, k+1}^{1 / 2}=\eta_{k}
$$

la deformazione (normalizzata) che dovri essere liberata dalla eventolale $(k+1)^{m a}$ replica affinché l'andamento risulti stazionario, si avrà

$$
x_{k+1}^{1 / 2}<x_{0, k+1}^{1 / 2}, \quad x_{k+1}^{1 / 2}=x_{0, k+1}^{1 / 2}, \quad x_{k+1}^{1 / 2}>x_{0, k+1}^{1 / 2}
$$

a seconda che il fenomeno si presenti in questa sucessiva eventuale replica, rispettivamente decrescente, stazionario oppure crescente.

Ciò vuol dire che à possibile prevedere il massimo e il minimo valore della deformazione che dovrà essere liberata dalla eventuale $(k+1)^{\text {ma }}$ replica, affinchi il processo assuma l'andamento decrescente o crescente rispettivamente, dato che il valore di $x_{o, k}$ i puo essere calcolato con $\mathrm{i}$ dati fino alla $k^{\text {ma }}$ replica, che somo noti.

In queste considemzioni si può anche introdure la magnitudo delle repliche.

Sia $H_{0}$ la magnitudo di ma scossa mica di energia $E_{0}$, cioc di una scossa di energia nguale alla somma delle energie delle scosse premonitorie e della scossa principale.

Dalla relazione contenuta nel secondo paragrafo si ha

$$
\mathrm{Log} E_{o}=b+a I_{o} \text {. }
$$

Poiché per una replica di energia $E_{k}$ risulta

$$
\mathrm{L} \operatorname{og} E_{k}-b+a M_{k} \text {. }
$$

Consegue

$$
\frac{a}{2}\left(M_{k}-M_{0}\right)=\log x_{k}^{1 / 2}
$$

da rui

$$
M_{k}=\| M_{o}+2 \mathrm{Log} x_{k}^{1 / 2} \text {. }
$$

Posto pertanto

$$
H_{o, k+1}=a H_{o}+2 \log x_{o, k+1}^{1 /,}
$$

che de una grandeyza nota, qualunque sia stato l'andamento del processo alla $k^{m a}$ replica, risulterà

$$
M_{k+1}<H_{o, k+1}, \quad M_{k+1}=H_{o, k+1}, \quad M_{k+1}>M I_{o, k+1}
$$

a seconda che alla $(k+1)^{\text {ma }}$ replica il processo si presenti rispettivamente decrescente, stazionario o rrescente.

Ia Tabella III chiarisce la questione. 


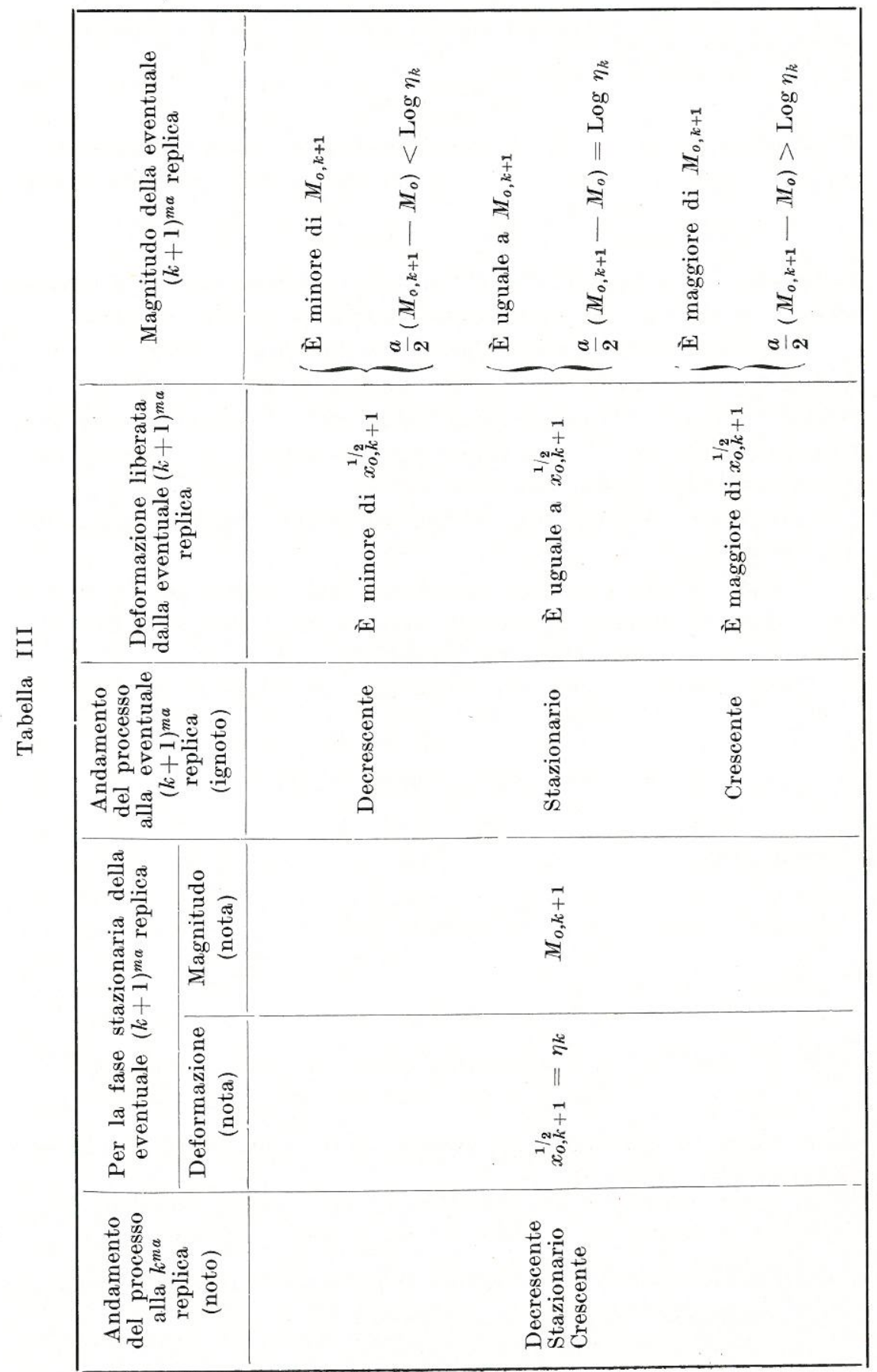


Il diagramma del rendimento attuale del processo delle repliche del terremoto siciliano del 15 Gennaio 1968 è riportato in Fig. 9.

Questo diagramma pone in evidenza che vi sono state soltanto tre fasi crescenti, ognuna costituita da una sola replica e corrispondenti alle repliche $k=3, \quad k=26, \quad k=42$.

\section{8. - Previsione dell'intervallo in cui potrà essere compresa \\ LA VARIAZIONE DEL RENDIMENTo atTuALE NEL PASSAGgio DA UNA REPLICA ALLA EVENTUALE SUCCESSIVA.}

Nel paragrafo precedente si è fatto vedere come l'introduzione del rendimento attuale del processo delle repliche consenta di risolvere tale processo in fasi decrescenti, stazionarie e crescenti e ciò in relazione alla variazione che detto parametro subisce da una replica alla successiva.

Questa variazione

$$
\Delta \eta_{k+1, k}=\frac{x_{k+1}^{1 / 2}\left(x_{k+1}^{1 / 2}-\eta_{k}\right)}{b_{k}+x_{k+1}^{1 / 2}}
$$

è una funzione continua della variabile $x_{k+1}$, definita nell'intervallo $0|-| 1$, e che può essere calcolata dopo la $k^{m a}$ replica, perchè sono noti i valori di $\eta_{k}$ e $b_{k}$.

Si vede facilmente che essa decresce al crescere di $x_{k+1}$, fino al valore minimo

$$
\Delta \eta_{m}=-\frac{x_{m}}{b_{k}}
$$

che assume per

$$
x_{k+1}=x_{m}
$$

con

$$
x_{m}=b_{k}^{2}\left(-1+\sqrt{1+\eta_{k} / b_{k}}\right)^{2}
$$

e poi cresce passando per lo zero quando

$$
x_{k+1}=x_{o, k+1}=\eta_{k}^{2}
$$

fino a raggiungere il valore più grande

$$
\Delta \eta_{M}=\frac{1-\eta_{k}}{1+b_{k}}
$$


che assume per

$$
x_{k+1}=1 .
$$

Dopo ogni replica si può quindi costruire, in funzione di $x_{k+1}$ o di $x_{k+1}^{1 / 2}$, il grafico dei valori che la variazione del rendimento attuale potrà assumere se successivamente si verificherà un'altra replica.

Nella Fig. 10 è riportato uno di questi grafici.

Esso è costruito con i dati fino alla replica venticinquesima e si riferisce alla eventuale replica ventiseiesima.

L'intervallo entro il quale può variare il rendimento attuale del processo delle repliche, nel passaggio del processo da una preplica alla eventuale successiva, può quindi essere previsto, poiché sussiste la limitazione

$$
-b_{k}\left(-1+\sqrt{1+\eta_{k} / b_{k}}\right)^{2} \leqslant \Delta \eta_{k+1, k} \leqslant \frac{1-\eta_{k}}{1+b_{k}} .
$$

Conviene peraltro considerare la variazione ridotta del rendimento attuale, il cui valore minimo prevedibile è definito dalla

$$
r_{m}=\frac{\Delta \eta_{m}}{\Delta \eta_{M}}
$$

da confrontare con l'eventuale analoga grandezza osservata

$$
r=\frac{\Delta \eta_{k+1, k}}{\Delta \eta_{M}}
$$

Risulterà sempre

$$
r_{m} \leqslant r \leqslant 1
$$

La grandezza $r_{m}$ può fornire indicazioni sulla tendenza del processo delle repliche a passare da una fase all'altra. Si supponga che alla $k^{m a}$ replica il processo risulti decrescente, ma che il valore di $r_{m}$ previsto per la eventuale $(k+1)^{m a}$ replica risulti elevato e prossimo allo zero. In questo caso è manifesta la possibilità che il processo alla eventuale $(k+1)^{m a}$ replica cambi fase, assumendo l'andamento crescente, col pericolo che l'energia liberata in onde elastiche da questa eventuale replica superi il valore previsto di $x_{o, k+1}$.

Viceversa accade se il processo risulta crescente, ma $r_{m}$ è molto piccolo. In questo caso è grande la possibilità che il processo passi da crescente a decrescente e che quindi la replica successiva liberi in onde elastiche un'energia inferiore al valore previsto di $x_{o, k+1}$. 


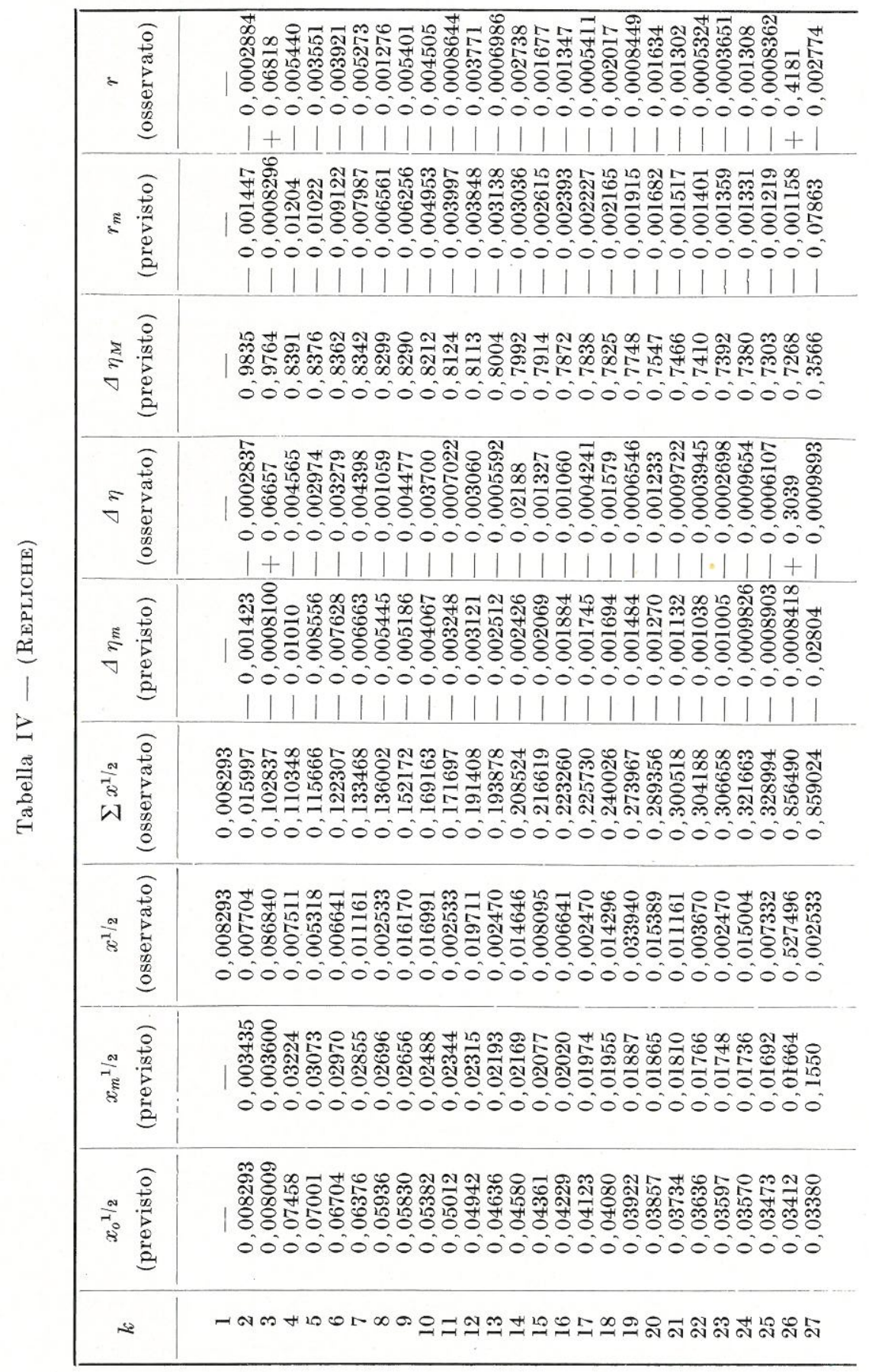




\begin{tabular}{|c|c|}
\hline 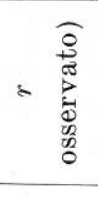 & 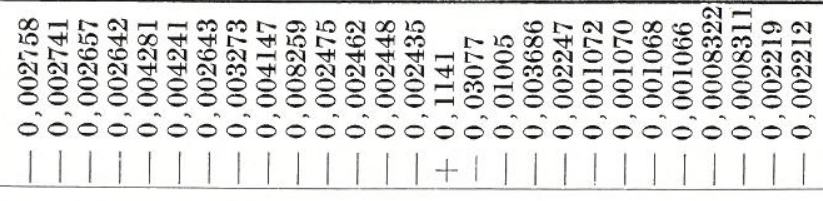 \\
\hline 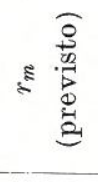 & 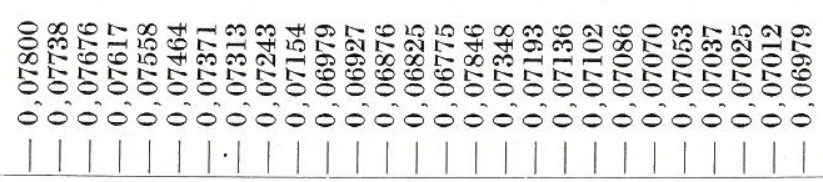 \\
\hline 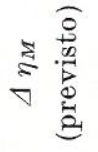 & 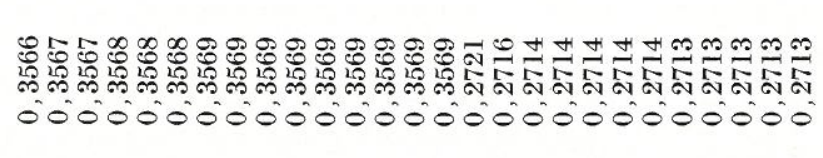 \\
\hline 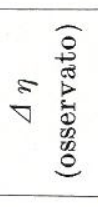 & 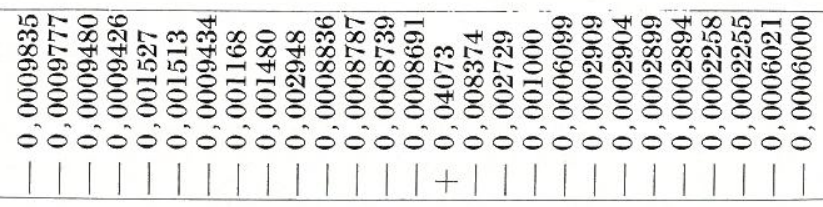 \\
\hline 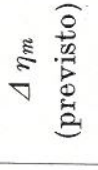 & 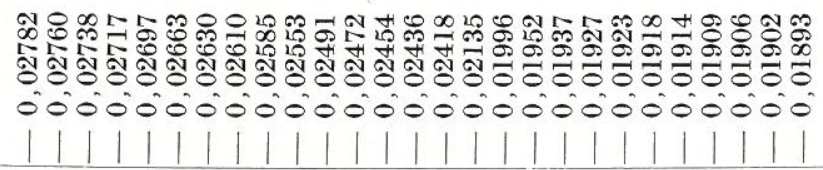 \\
\hline 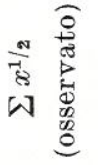 & 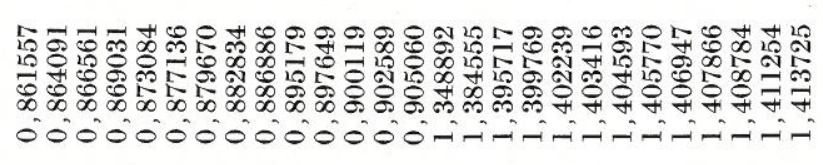 \\
\hline 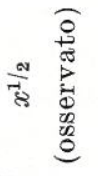 & 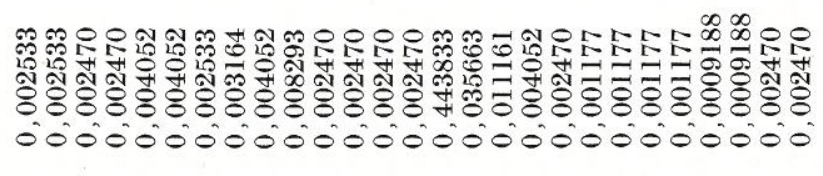 \\
\hline 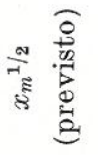 & 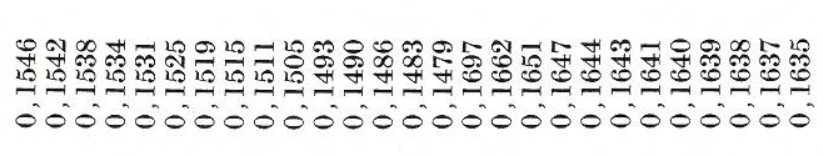 \\
\hline 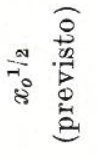 & 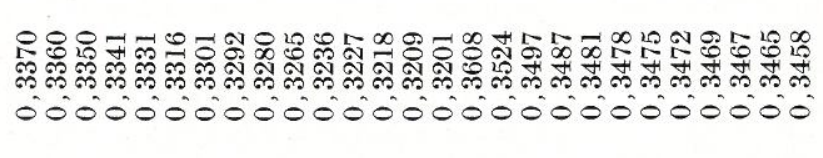 \\
\hline$\$$ & 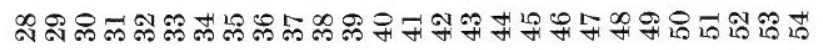 \\
\hline
\end{tabular}




\begin{tabular}{|c|c|}
\hline 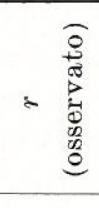 & 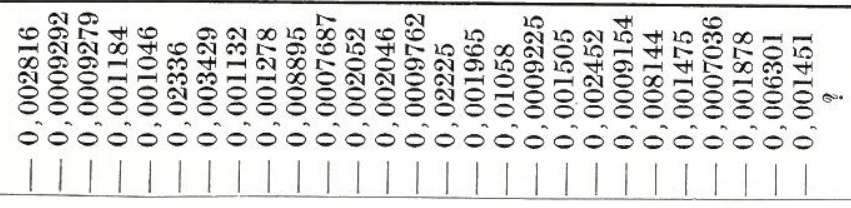 \\
\hline 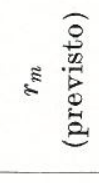 & 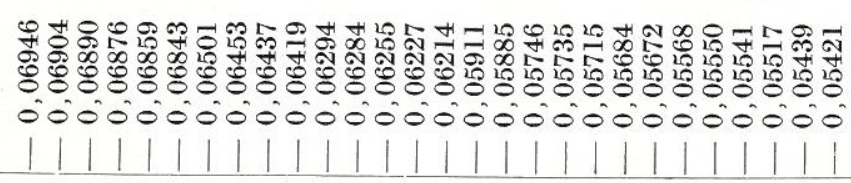 \\
\hline 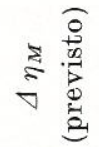 & 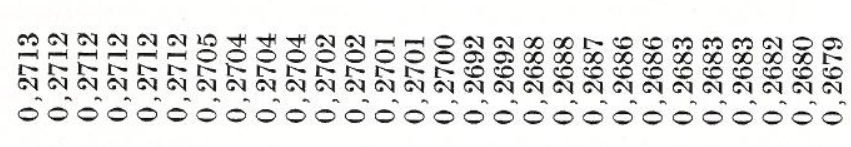 \\
\hline 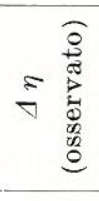 & 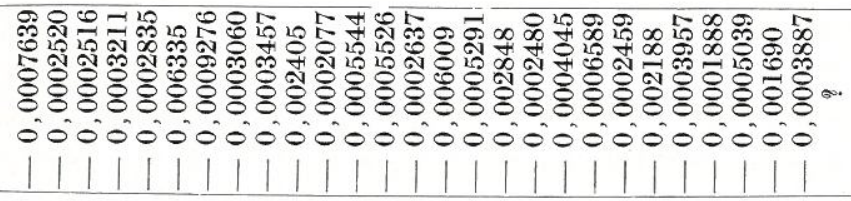 \\
\hline 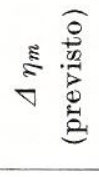 & 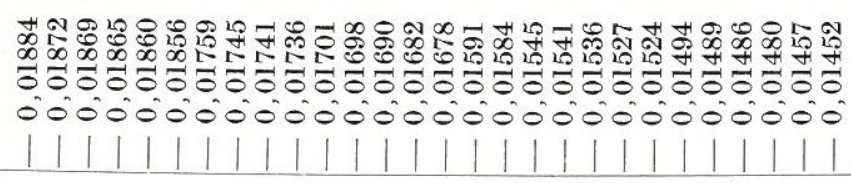 \\
\hline 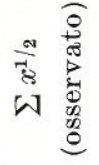 & 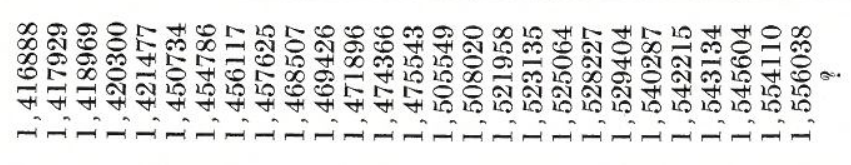 \\
\hline 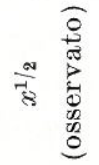 & 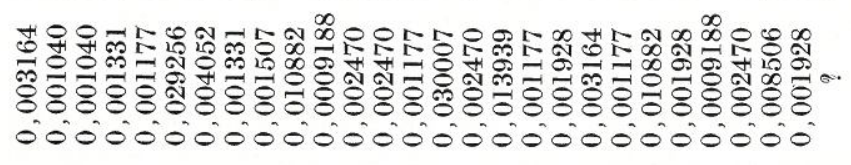 \\
\hline 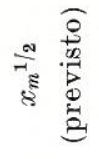 & 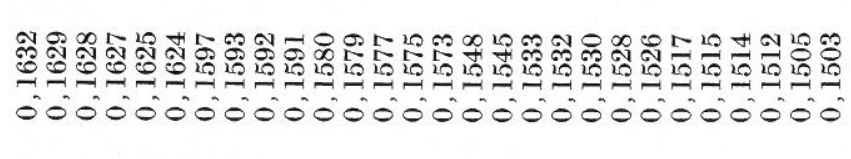 \\
\hline 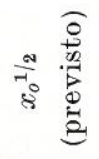 & 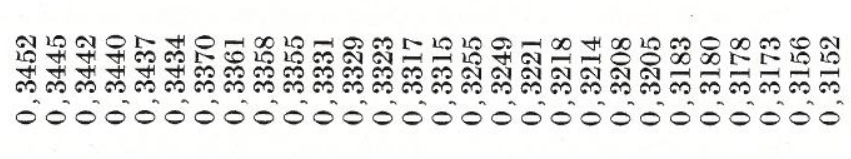 \\
\hline 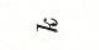 & 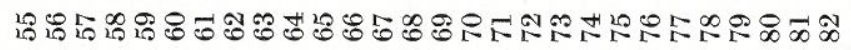 \\
\hline
\end{tabular}


Si osservino i diagrammi di Fig. 9 e Fig. 10, che si riferiscono al terremoto siciliano del 15 Gennaio 1968. Alla seconda replica il processo era decrescente, ma il valore previsto di $r_{m}$ per la eventuale successiva replica era elevato, rendendo manifesto il pericolo del passaggio del processo dalla fase decrescente alla fase crescente, come poi si verificò alla ter⿳a replica.

Alla terza replica $r_{m}$ diminui sensibilmente, facendo preverlere il ritorno del processo alla fase decrescente e ciò avvenne alla quarta repplica.

Dalla quarta replica alla venticinquesima il processo risulta decrescente, ma il valore previsto di $r_{m}$ ('resce progressivamente e alla venticinquesima replica i cosi vicino allo zero da far ritenere assai prossimo il cambiamento di fase del processo. (iò avveme alla ventiseiesima replicas.

Il valore di $r_{m}$ previsto per la eventuale replica ventisettesima risultio molto piccolo e infatti alla replica ventisettesima il processo ritorno alla fase decrescente, ma limitata da valori più elevati di $x_{o, k: 1}$ come era anche avvenuto dopo la terza replica.

Dalla replica ventisettesima il valore $r_{m}$ cresce abbastanza rapidamente, fino alla replica quarantaduesima, ma in questo caso il pericolo del passaggio alla fase crescente, avvenuto alla replica quarantatreesima, is meno manifesto.

Dalla replica quarantatreesima in poi il processo delle repliche e decrescente e i valori di $r_{m}$ si mantengono piuttosto piccoli.

In pratica l'elaborazione dei dati di Osservatorio ò stata eseguita, quando possibile, dopo ogni scossa e, in ogni altro caso, molto più frequentemente di quanto non avvenga in genere negli Osservatori sismologici in normale osservazione.

Nella Tabella IV sono contenuti i valori osservati e previsti delle grandezze relative alla serie delle repliche.

\section{9. - OSSERVhZIONI F CONCLUSiONI.}

Un fenomeno natumle può essere considerato in tre momenti, prima che il fenomeno si verifichi, durante la sua evoluzione a fenomeno conchuso.

In questo lavoro si i cercato di far vedere ciò che si può fare per tentare di porre sotto controllo un fenomeno sismico nel secondo momento e ron i dati di rui si puo disporre nel momento stesso. 
P. V. VALLE

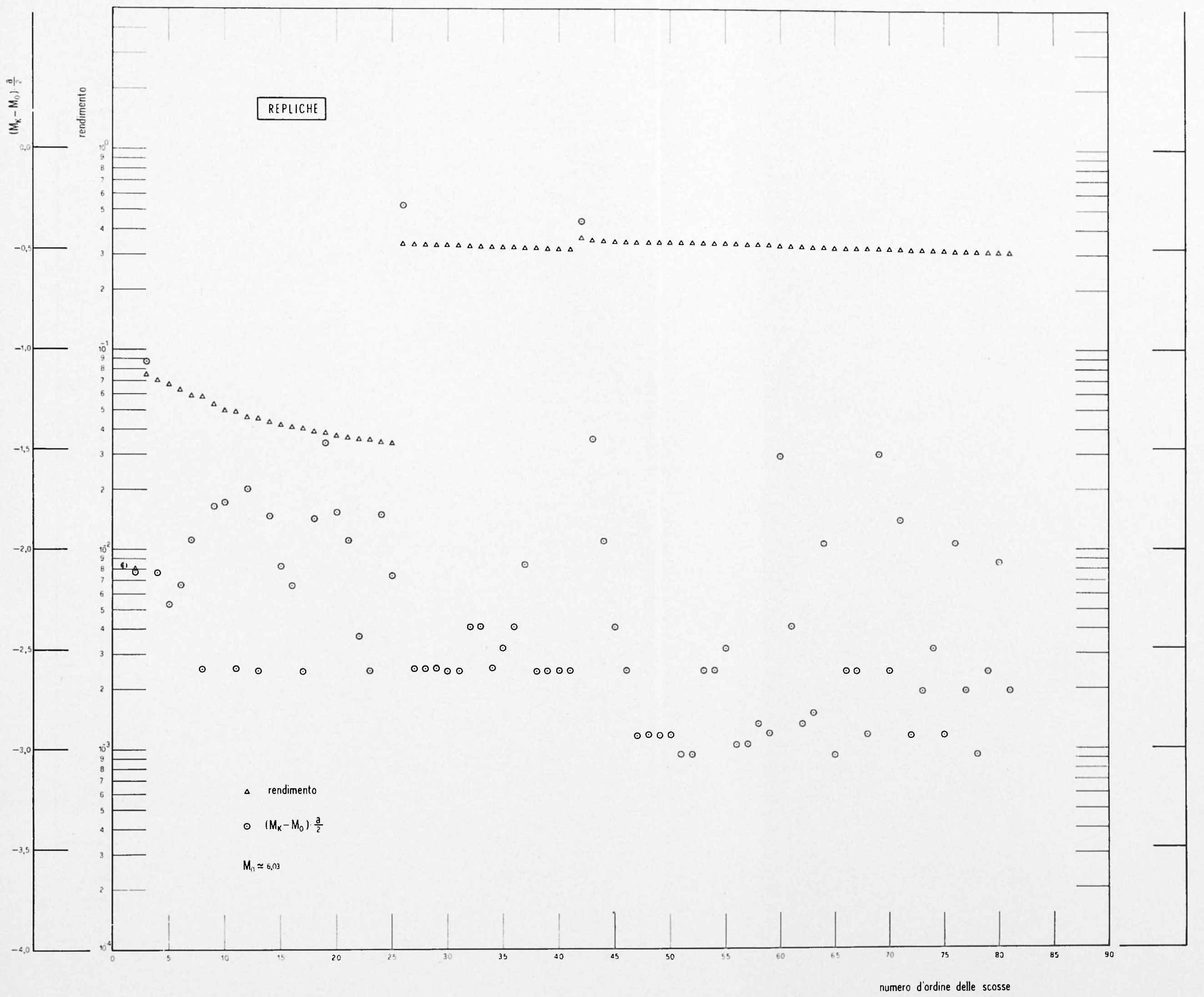

Fig. 9 - Terremoto siciliano del 1:j-1-1968. - Rendimento attuale dol processo delle r(pliche in funzione del relativo numero d'ordine. 


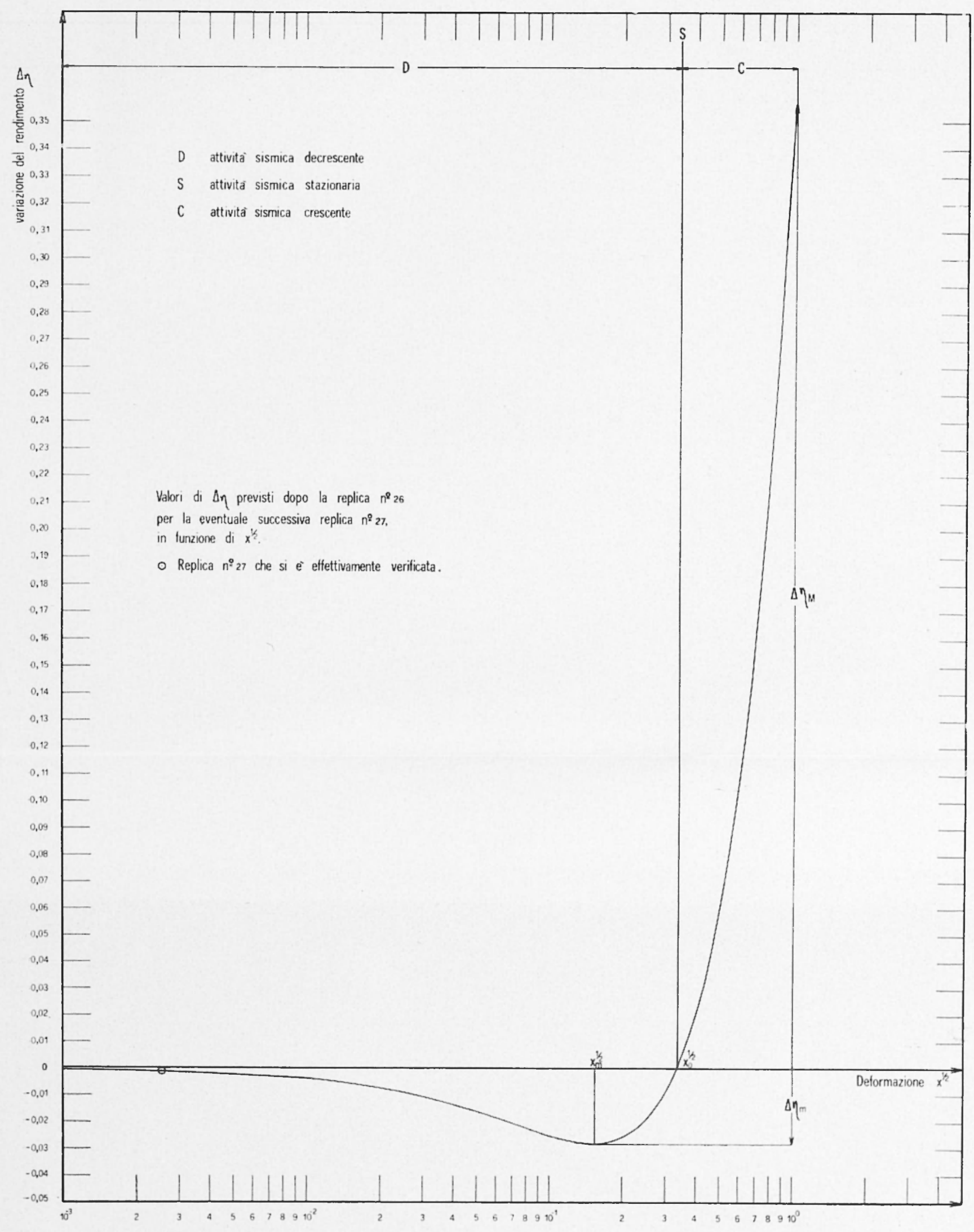

Fig. 10 - Terremoto siciliano del 15-1-1968. - Esempio di diagramma previsto per la variazione del rendimento attuale in funzione della deformazione. 
P. E. VALLE

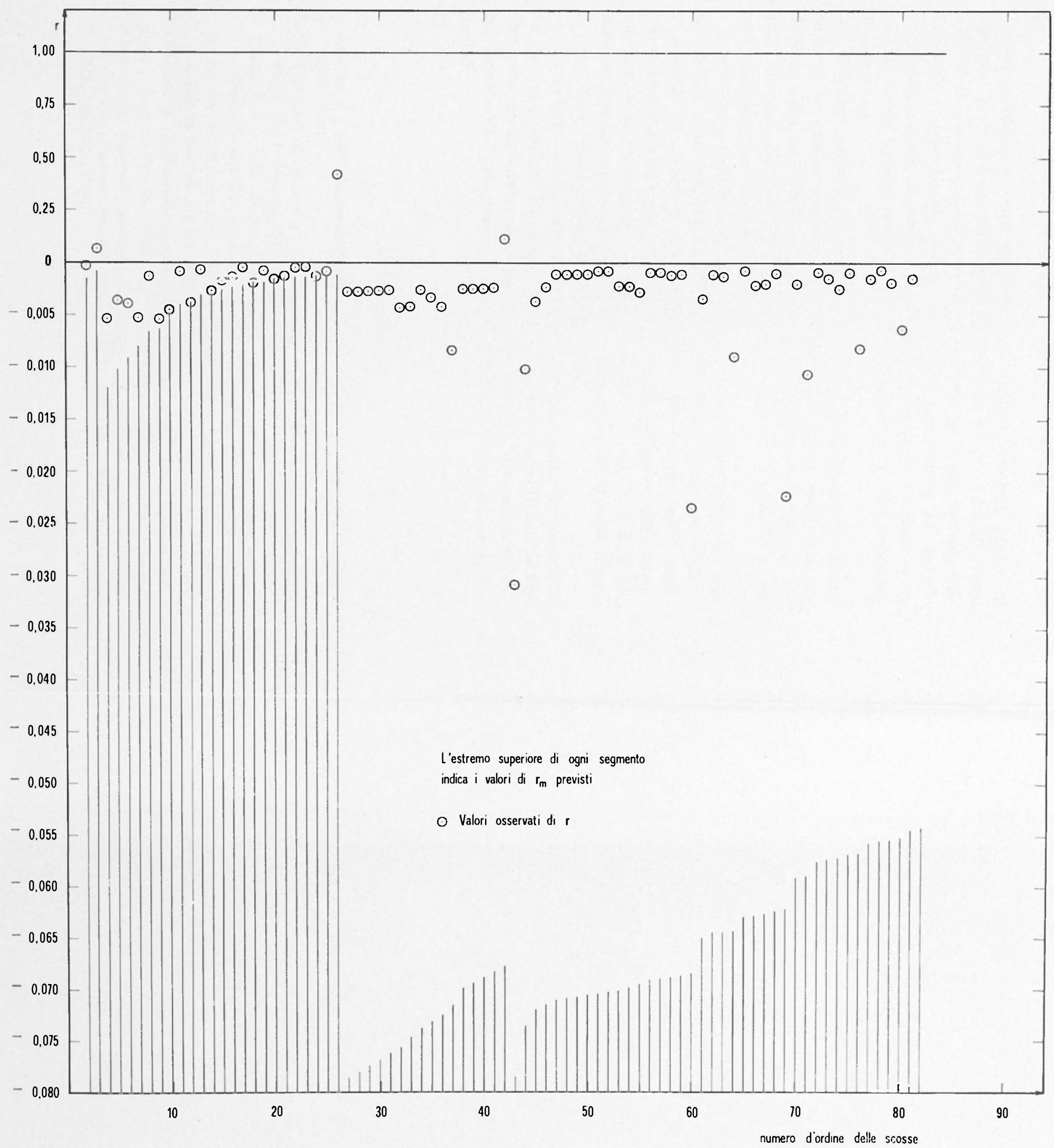

Fig. 11 - Terremoto siciliano del 15-1-1968. - Valori minimi previsti e valori osservati dalla variazione ridotta del rendimento attuale del processo delle repliche in funzione del loro numero d'ordine. 
Il problema è stato scisso in due problemi distinti. Liuno, di ranttere pratico, è costituito dalla ricerca della relazione fra gli eventi ron cui si realizza il fenomeno e i comispondenti eventi indicati dallorologio ronvenzionale, l'altro consiste nell'appliazione del metodo intrinseco, secondo il quale un fenomeno naturale va studiato in sè, rioè mediante i valori delle grandezze con coli gli eventi del fenomeno si concetetano.

Il primo problema is stato risolto enpiricamente con la costruzione di un grafico opportuno, per il secondo è stato continuato un discorso sulla teoria dell' e elastic rebound "iniziato da II. Benioff nel 1951, tenendo conto del carattere del fenomeno, che si manifesta con luna serie di eventi discreti (scosse).

Sebbene parecolie questioni non siano state sufficientemente approfondite, altre neppure menzionate e il presente lavoro costituisca in sostanza una relazione "sui generis" di quanto sembra avere qualche interesse ancom oggi, a fenomeno sismico siciliano praticamente concluso, si puo affermare che i gralici indicalti in Fig. 5, 7, 8, 9, 11, costruiti dumate un fenomeno sismico, consentono, entro rerti limiti, di controllare levolversi del fenomeno stesso.

Non risulta che tentativi analoghi siano stati fatti, ad eccezione del rontrollo dell'attivita sismical de Vuleano Sheveluche (Kamehatka) effettuato, prima e durante la grande eruzione del 12 Novembre $1964\left(^{9}\right)$, dall'Istituto di Vulcanologia Sovietico mediante il diagramma di $I$. Beniofi.

\section{BIBIAOGRAFIA}

(1) Bexiof H.. Earthquakes and rock creep. "Bull. Seism. Soc. Am, ". XLI. $31-62(1951)$.

(2) De Pavflurs M.-Marchlar L., Il periodo sismico della sicilia Occidentale inizialo il 14 Gemmaio 1968. "Amnali di Geofisiea ", XXI, 343-422 (1968).

(3) Valbe P. L.., sulla strullura della crosta terrestre nel Medilerraneo centrooccidentale e nell Adratico. "Annali di Geofisica ". IV. 399-409 (1951).

(1) Di Filippo D.-Marcela I.., Magniludo ed energia dei lerremoli in Italia. "Ammali di Geofisica ", III. 337-348 (1950).

(5) CAxcax A.. Sul periodo sismico iniziatosi il 24 A prile $1901 \mathrm{sul}$ lemilorio di Palombara Sabina. "Boll. Soc. Sism. Italiana ", VII. 169-193. (1901).

$\left.{ }^{6}\right)$ Cofalo M. Orogmafia, geologia e lellonica delle zone scosse dai lerremoli di Termini Imerese del Sellembre 1906. "Boll. Soc. Sism. Italiana ", XIII, $162-183(1908)$. 
(7) Cavasiso A., La frequenza delle repliche del grande terremoto nella Marsica. Valle del Liri del 1.3 Gennaio 191:j. "Boll. Soc. Sism. I1aliana ", XIX, $219-291$ (1915).

(") Greco N., Texi di laurea in corso di esecuzione.

$\left({ }^{y}\right)$ Tok arev P. I., The giant eruption of the Sheveluch Voleano on November 1:, 196.t, and its fore runners. Ac. Sciences U.S.S.R. (I.Z.V.) "Physics of the solid Earth ", 9, 572-579 (1967).

(10) 11 ancens. L... Breve relazione sull attivitì svolta dall' Istitudo Nazionale di Geofisica di Roma in occasione degli eventi sismici della Sicilia Occidentale. "A.G.I." Atti, XVII. 671.68I (1968). 